Sādhanā Vol. 40, Part 1, February 2015, pp. 51-74. (C) Indian Academy of Sciences

\title{
Automated adaptive sliding mode control scheme for a class of real complicated systems
}

\author{
M SHAHI $^{1, *}$ and A H MAZINAN ${ }^{2}$ \\ ${ }^{1}$ Department of Electrical Engineering, Birjand Branch, Islamic Azad University \\ (IAU), Birjand, Iran \\ ${ }^{2}$ Department of Control Engineering, Faculty of Electrical Engineering, \\ South Tehran Branch, Islamic Azad University (IAU), No. 209, North Iranshahr St., \\ P.O. Box 11365/4435, Tehran, Iran \\ e-mail: m.shahi@iaubir.ac.ir; mazinan@azad.ac.ir
}

MS received 14 December 2013; revised 14 September 2014; accepted 1 November 2014

\begin{abstract}
A class of real complicated systems, including chemical reactions, biological systems, information processing, laser systems, electrical circuits, information exchange, brain activities modelling, secure communication and other related ones can be presented through nonlinear and non-identical hyper-chaotic systems. The main goal of the present investigation is to synchronize two non-identical hyperchaotic master/slave systems, which are given as the models of the complicated systems, based on the realization of an efficient automated adaptive sliding mode control scheme. In the research presented here, the mentioned systems need to be dealt with through the proposed control scheme, since two non-identical systems are completely synchronized. In one such case, the whole of the chosen states of the master and slave systems should be coincided after a few time steps, as long as the effect of the external disturbance, uncertainty and unknown parameters could truly be ignored. Due to the fact that the investigated hyper-chaotic systems have taken into consideration as the representation of a number of complicated processes under mentioned external disturbance, uncertainty and unknown parameters, the traditional control approaches cannot actually be realized, in satisfactory manners. With this purpose, the proposed control scheme has been designed to cope with synchronization error, in a reasonable amount of time, in order to drive applicable hyper-chaotic systems. Consequently, the performance of the proposed control scheme is considered and verified through the numerical simulations.
\end{abstract}

Keywords. Complicated systems; efficient automated adaptive sliding mode control scheme; non-identical hyper-chaotic master/slave systems; synchronization error; numerical simulations.

*For correspondence 


\section{Introduction}

Nowadays, synchronization of hyper-chaotic systems is more applicable in different areas of engineering and other sciences and therefore the efficient results in this field could be useful. Contemporary research works are to develop techniques to hyper-chaotic systems, wherein the past two decades, chaos could have been taken into real consideration as a controversial issue in engineering, physics, mathematics and so on. This field is important to investigate due to the fact that a number of simple and complicated processes could be represented through these mathematical formulations. The whole of investigations that are now related to this area could significantly be increased for the purpose of presenting so many processes, in the satisfactory forms, in association with the corresponding control approaches. Generally, in terms of complexity, chaotic systems could be divided into three main categories including: normal chaotic systems, hyper-chaotic systems and finally spatio-temporal chaos systems. It is interesting to note that the greatest difference between chaotic systems and hyper-chaotic systems is that hyper-chaotic systems are taken as the generalized version of the previous one with at least two Lyapunov's positive exponents.

It should be noted that chaotic systems are known as nonlinear dynamic processes that are so sensitive to their initial conditions. It means that the slight changes in initial conditions in case of such systems can cause the corresponding great variation. In many applications, chaotic systems need to be replaced by the corresponding hyper-chaotic systems to represent some complicated processes, appropriately. Regarding the present concept, the butterfly effect is now known as a phenomenon that is a result of chaotic system's sensitivity to their initial conditions. It is shown that this phenomenon illustrates the great changes, since a slight change can be made in a chaotic system. In order to present the proposed approach, in an appropriate manner, a number of related works in this area are vastly considered in the following sub-section.

\subsection{Related works}

At first, a number of chaotic systems have been proposed by Lorenz (1963) and Rossler (1979). In the Lorenz research, finite systems of nonlinear differential equations are first designed, while their solutions are identified with trajectories in the phase space. In reality, systems with limited solutions are shown to possess bounded numerical solutions, since the system representing cellular convection is numerically solved. In the Rossler research, a simple four-variable oscillator containing one quadratic term produces a higher form of chaos with two directions of hyperbolic instability on the attractor. The present model difference equation is complicated, although its topology is truly simple. Guo (2012) investigate the stabilization of three-dimensional chaotic systems. Under the finite-time stability theory, a control law is realized to organize finite-time stabilization of three-dimensional chaotic systems (Guo 2012). An adaptive synchronous criteria for a general class of n-dimensional non-autonomous chaotic systems under linear and nonlinear feedback control approaches are considered. This idea can be generalized to a form for chaotic synchronization, as well. Based on stability theory on non-autonomous chaotic systems, some simple less conservative criteria for global asymptotic synchronization of the autonomous and non-autonomous chaotic systems are derived analytically. Subsequently, the results are applied to some typical chaotic systems such as the oscillators and the unified chaotic systems, while the numerical simulations are given to verify and also visualize the theoretical results (Ye \& Deng 2012).

An adaptive control approach for master-slave synchronization of two different fractionalorder chaotic systems under external disturbances was designed by Yin et al (2012). The bounds 
of the parameters, uncertainties and external disturbances were supposed to be unknown. Appropriate adaptive laws were suggested to tackle the system. The approach proposed here is realized to ensure the occurrence of the sliding motion and synchronization of two different fractionalorder systems (Yin et al 2012). The active control approach was realized for synchronization of two different pairs of fractional order systems under Lotka-Volterra chaotic system (Agrawal et al 2012). Here, the fractional derivative is described in Caputo sense. A salient feature of the present analysis is the revelation that the time for synchronization increases, as long as the system-pair approaches the integer order (Agrawal et al 2012). Abdullah (2013) dealt with the problems of chaos synchronization and secured communication in uncertain chaotic systems. The uncertain chaotic systems are subject to uncertainties modelling as well as a set of measurable system outputs. Here, two types of observations are suggested to achieve chaos synchronization and secure communication (Abdullah 2013). Kuntanapreeda \& Sangpet (2012) considered the problem of complete synchronization of chaotic systems with unknown parameters. An adaptive control approach in line with the feedback passivity approach was presented, since the convergence of the synchronization error was guaranteed. In one such case, the unified chaotic and hyper chaotic Lü systems were considered through numerical simulations (Kuntanapreeda \& Sangpet 2012). Chi-Ching (2012) presented an adaptive terminal control approach to achieve synchronization between two identical attractors which was related to a class of second-order chaotic system in the presence of system uncertainties, external disturbances and input nonlinearity. The proposed idea with adaptive feedback gains may compensate nonlinear dynamics of the synchronous error system (Chi-Ching 2012). Zhao (2012) investigated the adaptive Q-S synchronization of coupled chaotic systems with stochastic perturbation, delay and unknown parameters. The conditions for achieving Q-S synchronization of two stochastic chaotic systems were in line with the invariance principle of stochastic differential equation. The control laws and the corresponding parameter update laws were considered such that the stochastic Q-S synchronization of non-identical chaotic systems could be obtained (Zhao 2012). Razminia \& Baleanu (2013), have presented a new fractional order chaotic system to illustrate the behaviour such as two, three, and four scrolls. Synchronization of these systems is a problem either theoretically or practically. By realizing a sliding mode control methodology, a unidirectional coupling structure for the two chaotic systems has been synchronized (Razminia \& Baleanu 2013).

Yuan et al (2014) presented smart scheme for chaotic signal generation in a semiconductor ring laser under optical feedback. The chaotic oscillation can be generated by the partial reflection of the laser output. Time series, attracter and the power spectrum, in association with the Lyapunov exponent spectrum are considered. Synchronization of feedback-induced chaos was presented by optical injection, where it was acquired with suitable injection strength and detuning frequency (Yuan et al 2014). Yang et al (2014) dealt with the chaotic synchronization and chaos-based secure communication for chaotic systems. In this way, an augmented singular system was realized by introducing an augmented vector. Moreover, a second-order sliding mode observer was surveyed to get the exact estimates of output derivatives in a finite time. In line with estimates of states and output derivatives, a kind of algebraic unknown information recovery method was proposed (Yang et al 2014).

The mentioned research works as well as other unconsidered related ones have tried to present a number of new approaches in this area. The main difference between the approaches proposed in this research with respect to other corresponding ones are consideration of the specified realization of the control strategy and its results. A number of new adaptation laws have been investigated in accordance with the proposed control scheme. It should be noted that these factors are uniquely presented as the approach novelties. 
The rest of the present paper is organized as follows: the master/slave hyper-chaotic systems have been presented in section 2. The proposed adaptive sliding mode control scheme is given in section 3. Subsequently, the numerical simulation results and concluding remarks are given in sections 4 and 5 , respectively.

\section{The master/slave hyper-chaotic systems}

The master hyper-chaotic system is first described by the following

$$
\dot{x}_{i}(t)=m_{i}(x(t))+M_{i}(x(t)) \zeta+\Delta r_{i}(x(t))+d_{i}^{m}(t) ; i=1,2, \ldots, n,
$$

where $x(t)=\left[x_{1}, x_{2}, \ldots, x_{n}\right]^{T}$ is taken as the state vector, $m_{i}(x(t))$ is taken as $\mathrm{i}^{\text {th }}$ element of the continuous nonlinear function vector, $M_{i}(x(t))$ is taken as $\mathrm{i}^{\text {th }}$ row of $M(x(t))$ whose elements are the continuous nonlinear functions in association with $\zeta$ as the constant vector of unknown parameters, $\Delta r_{i}(x(t))$ is taken as $\mathrm{i}^{\text {th }}$ row of $\Delta r(x(t))=$ $\left[\Delta r_{1}(x(t)), \Delta r_{2}(x(t)), \ldots, \Delta r_{n}(x(t))\right]^{T}$, i.e., the vector of unknown uncertainties and $d_{i}^{m}(t)$ is finally taken as ith row of $d^{m}(t)=\left[d_{1}^{m}(t), d_{2}^{m}(t), \ldots, d_{n}^{m}(t)\right]^{T}$, i.e., the external disturbances. And the slave hyper-chaotic system is then described as

$$
\dot{y}_{i}(t)=t_{i}(y(t))+T_{i}(y(t)) \Gamma+\Delta R_{i}(y(t))+d_{i}^{S}(t)+u_{i}(t) ; i=1,2, \ldots, n,
$$

where $y(t)=\left[y_{1}, y_{2}, \ldots, y_{n}\right]^{T}$ is taken as the state vector, $t_{i}(y(t))$ is taken as $\mathrm{i}^{\text {th }}$ element of the vector of the nonlinear function and it is assumed to be continuous, $T_{i}(y(t))$ is taken as $\mathrm{i}^{\text {th }}$ row of $T(y(t))$ whose elements are the continuous nonlinear functions in association with $\Gamma$ as the constant vector of unknown parameter, $u_{i}(t)$ is taken as $\mathrm{i}^{\text {th }}$ desired control action, $\Delta R_{i}(y(t))$ is taken as $i^{\text {th }}$ element of $\Delta R(y(t))=\left[\Delta R_{1}(y(t)), \Delta R_{2}(y(t)), \ldots, \Delta R_{n}(y(t))\right]^{T}$, i.e., the vector of unknown uncertainties and $d_{i}^{s}(t)$ is finally taken as $\mathrm{i}^{\text {th }}$ element of $d^{s}(t)=$ $\left[d_{1}^{s}(t), d_{2}^{s}(t), \ldots, d_{n}^{s}(t)\right]^{T}$, i.e. the external disturbances.

It should be noted that a synchronization scheme, which is organized based on two hyperchaotic systems, as the master and the slave systems, is presented. Figure 1 illustrates these hyper-chaotic systems as well as the proposed control approach. The main contribution of the control approach, which is realized here, is to make synchronization. It means that the states of the master system should be followed by the corresponding states of the slave system, which are all in the presence of uncertainties, external disturbances and fully unknown parameters.

Definition 1. The chaos synchronization error is defined as $e(t)=y(t)-x(t)$, and the control goal is to realize control action; $u(t)$, so that the trajectory of the slave system; Eq. (2), can approach the maser system asymptotically; Eq. (1), under different initial conditions. In another

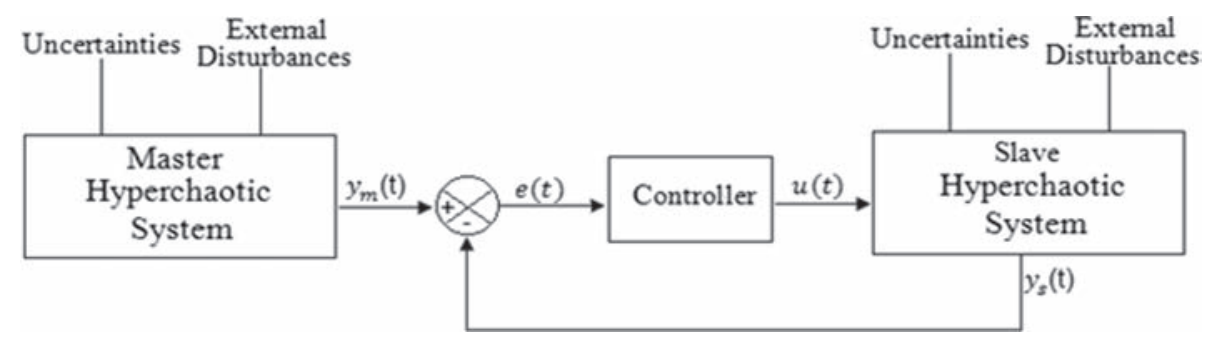

Figure 1. Schematic of the hyper-chaotic systems with its control approach. 
words, synchronization error between the present systems; Eqs. (1) and (2), in the presence of the uncertainties, external disturbances and unknown parameters needs to satisfy $\lim _{t \rightarrow \infty}\|e(t)\|=0$.

Assumption 1. The parameters $\omega_{i}^{m}$ and $\omega_{i}^{s} ; i=1,2, \ldots, n$ are taken as the maximum estimation of $\left|\Delta r_{i}(x(t))\right|$ and $\left|\Delta R_{i}(y(t))\right|$ as the uncertainty models in the systems; Eqs. (1) and (2), respectively. It means that the models uncertainties are assumed to be bounded as

$$
\left|\Delta r_{i}(x(t))\right|<\omega_{i}^{m} \quad \text { and } \quad\left|\Delta R_{i}(y(t))\right|<\omega_{i}^{s}, \quad i=1,2, \ldots, n .
$$

Therefore, one can conclude that

$$
\left|\Delta R_{i}(y(t))-\Delta r_{i}(x(t))\right|<\omega_{i}, \quad i=1,2, \ldots, n
$$

Assumption 2. External disturbances are supposed to be bounded, where $\beta_{i}^{m}$ and $\beta_{i}^{s} ; i=$ $1,2, \ldots, n$ are taken as the maximum estimation of $d_{i}^{s}(t)$ and $d_{i}^{m}(t)$, i.e., the model external disturbances, in the present systems; Eqs. (1) and (2), respectively. Therefore, there are two appropriate positive constants, i.e., $\beta_{i}^{\mathrm{m}}$ and $\beta_{i}^{\mathrm{s}}$ such that

$$
\left|d_{i}^{m}(t)\right|<\beta_{i}^{m} \quad \text { and } \quad\left|d_{i}^{s}(t)\right|<\beta_{i}^{s}, \quad i=1,2, \ldots, n \text {. }
$$

As a result, one can obtain as

$$
\left|d_{i}^{s}(t)-d_{i}^{m}(t)\right|<\beta_{i}, \quad i=1,2, \ldots, n .
$$

Assumption 3. The parameters $\omega_{i}$ and $\beta_{i} ; i=1,2, \ldots, n$ are unknown and positive constants. Let us remember $e(t)=y(t)-x(t)$ as the synchronization error. Then, the dynamics of the system error are directly calculated by subtracting Eqs. (1) and (2). Consequently, the error dynamics are acquired through Eq. (7), i.e.,

$$
\begin{aligned}
\dot{e}_{i}(t)= & t_{i}(y(t))+T_{i}(y(t)) \Gamma+\Delta R_{i}(y(t))+d_{i}^{s}(t)-m_{i}(x(t))-M_{i}(x(t)) \zeta \\
& -\Delta r_{i}(x(t))-d_{i}^{m}(t)+u_{i}(t) ; i=1,2, \ldots, n .
\end{aligned}
$$

\section{The proposed adaptive sliding mode control scheme}

The sliding mode control scheme which is outperforming through adaptive laws is investigated in the present research to act as the controller. In case of the sliding mode control, it is obvious that so many researchers realize this control technique as the robust method. The main reason of realizing the present method is to make the robust control reliable against a number of problems such as uncertainty and disturbance. In case of the adaptive control, it is also obvious that there are some suitable methods to overcome the uncertainties. By integrating the adaptive control and the sliding mode control, the efficient and applicable robust control approach against uncertainties and disturbances could be designed. While the system remains in the sliding surface in the presence of uncertainty, disturbance and unknown parameters, a number of efficient adaptation laws should play important role in guaranteeing the system performance. As a result, to propose the adaptive sliding mode control approach, two steps are needed. The first step of the sliding mode control realization is to indicate the suitable sliding surface, while the second one is to indicate the appropriate control law, in order to move the system, consistently, towards the indicated surface for the purpose of remaining there. Moreover, the control approach should 
be designed such that the system state trajectories are forced to the sliding surface for staying there. Therefore, the following sliding surfaces need to be first chosen by Eq. (8), i.e.,

$$
s_{i}(t)=\lambda_{i} e_{i} ; i=1,2, \ldots, n,
$$

where $s(t)=\left[s_{1}, s_{2}, \ldots, s_{n}\right]^{T} ; s_{i}(t) \in R$ is defined and the parameters $\lambda_{i}, i=1,2, \ldots, n$ are taken as the positive constants. In addition, the next step is to design the control actions; $u_{i}(t)$; and the realization of the proposed control approach is to guarantee that the system error trajectories reach to the desirable sliding surface, i.e., $s(t)=\dot{s}(t)=0$. To satisfy the reaching condition $s \dot{s}<0$, in a finite time for staying there forever. To ensure the occurrence of the sliding motion, the following adaptive sliding mode control actions are investigated

$$
\begin{aligned}
u_{i}(t)= & m_{i}(x(t))-t_{i}(y(t))+M_{i}(x(t)) \hat{\zeta}-T_{i}(y(t)) \hat{\Gamma}-\Delta R_{i}(y(t))-d_{i}^{s}(t) \\
& +\Delta r_{i}(x(t))+d_{i}^{m}(t)-u_{q} ; i=1,2, \ldots, n,
\end{aligned}
$$

where $\hat{\zeta}, \hat{\Gamma}, \hat{\omega}_{i}$ and $\hat{\beta}_{i}$ are all taken as the estimations of $\zeta, \Gamma, \omega_{i}$ and $\beta_{i}$, respectively. For completing the present procedure for the purpose of realizing the appropriate control approach to be able to deal with the system trajectories into the sliding surface, the reaching law is specified as $u_{q}=k_{i} \operatorname{sgn}\left(s_{i}\right)$. Here, the switching gain; $k_{i}>0$, could be a positive constant. Now, the proposed adaptive sliding mode control approach is resulted by the following

$$
\begin{aligned}
u_{i}(t)= & m_{i}(x(t))-t_{i}(y(t))+M_{i}(x(t)) \hat{\zeta}-T_{i}(y(t)) \hat{\Gamma}-\Delta R_{i}(y(t))-d_{i}^{s}(t) \\
& +\Delta r_{i}(x(t))+d_{i}^{m}-k_{i} \operatorname{sgn}\left(s_{i}\right) ; i=1,2, \ldots, n .
\end{aligned}
$$

Afterwards by taking the assumptions 1 and 2 into account while the trajectories of the chaotic system in association with the unstructured uncertainties and the disturbances are all bounded, the control action is resulted by

$$
\begin{aligned}
u_{i}(t)= & m_{i}(x(t))-t_{i}(y(t))+M_{i}(x(t)) \hat{\zeta}-T_{i}(y(t)) \hat{\Gamma}-\left(\hat{\omega}_{i}+\hat{\beta}_{i}\right) \operatorname{sgn}\left(s_{i}\right) \\
& -k_{i} \operatorname{sgn}\left(s_{i}\right) ; i=1,2, \ldots, n,
\end{aligned}
$$

where the following adaptation laws are taken

$$
\begin{cases}\dot{\hat{\beta}}_{i}(t)=\lambda_{i}\left|s_{i}\right| ; & \hat{\beta}_{i}(0)=\hat{\beta}_{i 0} \\ \dot{\hat{\omega}}_{i}(t)=\lambda_{i}\left|s_{i}\right| ; & \hat{\omega}_{i}(0)=\hat{\omega}_{i 0} \\ \dot{\hat{\Gamma}}(t)=[T(y(t))]^{T} \eta ; & \hat{\Gamma}(0)=\hat{\Gamma}_{0} \\ \dot{\hat{\zeta}}(t)=-[M(x(t))]^{T} \eta ; & \hat{\zeta}(0)=\hat{\zeta}_{0} .\end{cases}
$$

Here, $\eta=\left[\lambda_{1} s_{1}, \lambda_{2} s_{2}, \ldots, \lambda_{n} s_{n}\right]^{T}$ is assumed and $\lambda$ is taken as a positive constant. In addition, $\hat{\zeta}_{0}, \hat{\Gamma}_{0}, \hat{\omega}_{i 0}$ and $\hat{\beta}_{i 0}$ are all taken as the initial values of the present parameters, i.e., $\hat{\zeta}, \hat{\Gamma}, \hat{\omega}_{i}$ and $\hat{\beta}_{i}$, respectively,

Proof. In order to consider the coverage of the present adaptation laws; (Eq. (12)) in association with the proposed control scheme, the following Lyapunov function is first chosen as a candidate, i.e.,

$$
V(t)=\frac{1}{2} \sum_{i=1}^{n}\left[s_{i}^{2}+\left(\hat{\omega}_{i}-\omega_{i}\right)^{2}+\left(\hat{\beta}_{i}-\beta_{i}\right)^{2}\right]+\frac{1}{2}\|\hat{\zeta}-\zeta\|^{2}+\frac{1}{2}\|\hat{\Gamma}-\Gamma\|^{2}
$$


while assuming $s_{i}(t)=\lambda_{i} e_{i} ; i=1,2, \ldots, n$, the derivation of time $t$ of the present Lyapunov function could be resulted as

$$
\dot{V}(t)=\sum_{i=1}^{n}\left[s_{i} \dot{s}_{i}+\left(\hat{\omega}_{i}-\omega_{i}\right) \dot{\hat{\omega}}_{i}+\left(\hat{\beta}_{i}-\beta_{i}\right) \dot{\hat{\beta}}_{i}\right]+(\hat{\zeta}-\zeta)^{T} \dot{\hat{\zeta}}+(\hat{\Gamma}-\Gamma)^{T} \dot{\hat{\Gamma}}
$$

By using Eq. (7) in Eq. (14), the results could be apparent by the following

$$
\begin{aligned}
\dot{V}(t)= & \sum_{i=1}^{n}\left[\lambda _ { i } s _ { i } \left(-m_{i}(x(t))-M_{i}(x(t)) \zeta-\Delta r_{i}(x(t))-d_{i}^{m}(t)+t_{i}(y(t))\right.\right. \\
& \left.\left.+T_{i}(y(t)) \Gamma+\Delta R_{i}(y(t))+d_{i}^{S}(t)+u_{i}(t)\right)+\left(\hat{\omega}_{i}-\omega_{i}\right) \dot{\hat{\omega}}_{i}+\left(\hat{\beta}_{i}-\beta_{i}\right) \dot{\hat{\beta}}_{i}\right] \\
& +(\hat{\zeta}-\zeta)^{T} \dot{\hat{\zeta}}+(\hat{\Gamma}-\Gamma)^{T} \dot{\hat{\Gamma}} .
\end{aligned}
$$

Now, by using the adaptation law; Eq. (12), the following relation yields

$$
\begin{aligned}
\dot{V}(t)= & \sum_{i=1}^{n}\left[\lambda _ { i } s _ { i } \left(-m_{i}(x(t))-M_{i}(x(t)) \zeta-\Delta r_{i}(x(t))-d_{i}^{m}(t)+t_{i}(y(t))\right.\right. \\
& \left.+T_{i}(y(t)) \Gamma+\Delta R_{i}(y(t))+d_{i}^{s}(t)\right)+u_{i}(t) \lambda_{i} s_{i}+\left(\hat{\omega}_{i}-\omega_{i}\right) \lambda_{i}\left|s_{i}\right| \\
& \left.+\left(\hat{\beta}_{i}-\beta_{i}\right) \lambda_{i}\left|s_{i}\right|\right]-(\hat{\zeta}-\zeta)^{T}[M(x(t))]^{T} \eta+(\hat{\Gamma}-\Gamma)^{T}[T(y(t))]^{T} \eta .
\end{aligned}
$$

By defining the following

$$
\left\{\begin{array}{l}
\sum_{i=1}^{n} \lambda_{i} s_{i} M_{i}(x(t)) \zeta=\zeta^{T}[M(x(t))]^{T} \eta \\
\sum_{i=1}^{n} \lambda_{i} s_{i} T_{i}(y(t)) \Gamma=\Gamma^{T}[T(y(t))]^{T} \eta
\end{array}\right.
$$

Eq. (16) is easily given in the form of Eq. (18), i.e.,

$$
\begin{aligned}
\dot{V}(t)= & \sum_{i=1}^{n}\left[\lambda_{i} s_{i}\left(-m_{i}(x(t))-\Delta r_{i}(x(t))-d_{i}^{m}(t)+t_{i}(y(t))+\Delta R_{i}(y(t))+d_{i}^{s}(t)\right)\right. \\
& \left.+s_{i} \lambda_{i} u_{i}(t)+\left(\hat{\omega}_{i}-\omega_{i}\right) \lambda_{i}\left|s_{i}\right|+\left(\hat{\beta}_{i}-\beta_{i}\right) \lambda_{i}\left|s_{i}\right|\right]-\hat{\zeta}^{T}[M(x(t))]^{T} \eta \\
& +\hat{\Gamma}^{T}[T(y(t))]^{T} \eta .
\end{aligned}
$$

By inserting Eq. (11) in Eq. (18), Eq. (19) yields as

$$
\begin{aligned}
\dot{V}(t)= & \sum_{i=1}^{n}\left[\lambda_{i} s_{i}\left(-m_{i}(x(t))-\Delta r_{i}(x(t))-d_{i}^{m}(t)+t_{i}(y(t))+\Delta R_{i}(y(t))+d_{i}^{s}(t)\right)\right. \\
& +s_{i} \lambda_{i}\left(m_{i}(x(t))-t_{i}(y(t))+M_{i}(x(t)) \hat{\zeta}-T_{i}(y(t)) \hat{\Gamma}-\left(\hat{\omega}_{i}+\hat{\beta}_{i}\right) \operatorname{sgn}\left(s_{i}\right)\right. \\
& \left.\left.-k_{i} \operatorname{sgn}\left(s_{i}\right)\right)+\left(\hat{\omega}_{i}-\omega_{i}\right) \lambda_{i}\left|s_{i}\right|+\left(\hat{\beta}_{i}-\beta_{i}\right) \lambda_{i}\left|s_{i}\right|\right]-\hat{\zeta}^{T}[M(x(t))]^{T} \eta \\
& +\hat{\Gamma}^{T}[T(y(t))]^{T} \eta .
\end{aligned}
$$

Now, it is clear to note

$$
\begin{aligned}
\dot{V}(t) \leq & \sum_{i=1}^{n}\left[\lambda_{i}\left|s_{i}\right|\left(\left|\Delta R_{i}(y(t))-\Delta r_{i}(x(t))\right|+\left|d_{i}^{s}(t)-d_{i}^{m}(t)\right|\right)+s_{i} \lambda_{i}\left(M_{i}(x(t)) \hat{\zeta}\right.\right. \\
& \left.-T_{i}(y(t)) \hat{\Gamma}-\left(\hat{\omega}_{i}+\hat{\beta}_{i}\right) \operatorname{sgn}\left(s_{i}\right)-k_{i} \operatorname{sgn}\left(s_{i}\right)\right)+\left(\hat{\omega}_{i}-\omega_{i}\right) \lambda_{i}\left|s_{i}\right| \\
& \left.+\left(\hat{\beta}_{i}-\beta_{i}\right) \lambda_{i}\left|s_{i}\right|\right]-\hat{\zeta}^{T}[M(x(t))]^{T} \eta+\hat{\Gamma}^{T}[T(y(t))]^{T} \eta .
\end{aligned}
$$




$$
\begin{aligned}
\dot{V}(t) \leq & \sum_{i=1}^{n}\left[\lambda_{i}\left|s_{i}\right|\left(\omega_{i}+\beta_{i}\right)+s_{i} \lambda_{i}\left(M_{i}(x(t)) \hat{\zeta}-T_{i}(y(t)) \hat{\Gamma}-\left(\hat{\omega}_{i}+\hat{\beta}_{i}\right) \operatorname{sgn}\left(s_{i}\right)\right.\right. \\
& \left.\left.-k_{i} \operatorname{sgn}\left(s_{i}\right)\right)+\left(\hat{\omega}_{i}-\omega_{i}\right) \lambda_{i}\left|s_{i}\right|+\left(\hat{\beta}_{i}-\beta_{i}\right) \lambda_{i}\left|s_{i}\right|\right]-\hat{\zeta}^{T}[M(x(t))]^{T} \eta \\
& +\hat{\Gamma}^{T}[T(y(t))]^{T} \eta
\end{aligned}
$$

It is easy to rewrite Eq. (21) in the form of Eq. (22), i.e.,

$$
\begin{aligned}
\dot{V}(t) \leq & \sum_{i=1}^{n}\left[s_{i} \lambda_{i}\left(M_{i}(x(t)) \hat{\zeta}-T_{i}(y(t)) \hat{\Gamma}-\left(\hat{\omega}_{i}+\hat{\beta}_{i}\right) \operatorname{sgn}\left(s_{i}\right)-k_{i} \operatorname{sgn}\left(s_{i}\right)\right)\right. \\
& \left.+\hat{\omega}_{i} \lambda_{i}\left|s_{i}\right|+\hat{\beta}_{i} \lambda_{i}\left|s_{i}\right|\right]-\hat{\zeta}^{T}[M(x(t))]^{T} \eta+\hat{\Gamma}^{T}[T(y(t))]^{T} \eta .
\end{aligned}
$$

By presenting Eq. (23) and also defining $\operatorname{sgn}\left(s_{i}\right)=\left|s_{i}\right| /\left|s_{i}\right|=\lambda_{i}\left|s_{i}\right|$, Eq. (24) could then be deduced by

$$
\begin{gathered}
\left\{\begin{array}{l}
\sum_{i=1}^{n} S_{i} \lambda_{i} M_{i}(x(t)) \hat{\zeta}=\hat{\zeta}^{T}[M(x(t))]^{T} \eta \\
\sum_{i=1}^{n} S_{i} \lambda_{i} T_{i}(y(t)) \hat{\Gamma}=\hat{\Gamma}^{T}[T(y(t))]^{T} \eta
\end{array}\right. \\
\dot{V}(t) \leq \sum_{i=1}^{n}\left[-s_{i} \lambda_{i}\left(\left(\hat{\omega}_{i}+\hat{\beta}_{i}\right) \operatorname{sgn}\left(s_{i}\right)\right)+\left(\hat{\omega}_{i} \lambda_{i}\left|s_{i}\right|+\hat{\beta}_{i} \lambda_{i}\left|s_{i}\right|\right)-\left(k_{i} \lambda_{i}\left|s_{i}\right|\right)\right] .
\end{gathered}
$$

And therefore it is obvious to note

$$
\dot{V}(t) \leq \sum_{i=1}^{n}\left(-\left(\hat{\omega}_{i}+\hat{\beta}_{i}\right) \lambda_{i}\left|s_{i}\right|+\hat{\omega}_{i} \lambda_{i}\left|s_{i}\right|+\hat{\beta}_{i} \lambda_{i}\left|s_{i}\right|-k_{i} \lambda_{i}\left|s_{i}\right|\right) .
$$

In this way, Eq. (25) is easily written through Eq. (26), i.e.,

$$
\dot{V}(t) \leq \sum_{i=1}^{n}-k_{i} \lambda_{i}\left|s_{i}\right|
$$

Now, to analyse the requirement for the $\dot{V}(t)$ to be negative semi definite, let us rewrite the last result, which is now investigated through Eq. (25), in the form of Eq. (27), since Eq. (8) is taken into consideration

$$
\dot{V}(t) \leq \sum_{i=1}^{n}-k_{i} \lambda_{i}^{2}\left|e_{i}\right|
$$

In order to guarantee the $\dot{V}(t)$ to be negative semi definite, the values regarding $k_{i} ; i=$ $1,2, \ldots, n$ should positively be chosen, i.e., $k_{i}>0$. It means that the parameters $k_{i}$ must be taken as positive constants to guarantee that the coverage of the adaptation law is desirable. Due to the fact that the $\dot{V}(t)$ is now resulted as negative semi definite, in such a case, the reaching condition, i.e., $\dot{s}(t) s(t)<0$ based on the Lyapunov-stability theorem has been correspondingly approved.

Theorem 1. Consider the error dynamics; Eq. (7), and the sliding surface vector; Eq. (8). Now, the trajectories of the system under the control action; Eq. (11), in association with the adaptation laws; Eq. (12), can be into the sliding surface, i.e., $s(t)=0$. This theorem can be proved through the Lyapunov theorem.

Proof. Let us define $\mu_{i}=k_{i} \lambda_{i} ; i=1,2, \ldots, n$ and $|s|=\left[\left|s_{1}\right|,\left|s_{2}\right|, \ldots,\left|s_{n}\right|\right]^{T}$. Moreover, it is needed to assume $\mu=\left[\mu_{1}, \mu_{2}, \ldots, \mu_{n}\right]^{T}>0$. Regarding the procedure of investigation from Eq. (7) to Eq. (27) in line with the Lyapunov theorem, by using $\mu|s|=\sigma(t) \geq 0$, the last result can be rewritten as

$$
\dot{V}(t)=-\mu|s| \leq 0
$$


Due to the fact that the present investigation results i.e., $\dot{V}(t) \leq 0$ and $V(0)-V(t) \geq 0$ are acquired and also $\lim _{t \rightarrow \infty} \int_{0}^{\mathrm{t}} \sigma(\lambda) \mathrm{d} \lambda$ is limited, Eq. (29) yields by the following

$$
\lim _{t \rightarrow \infty} \int_{0}^{t} \sigma(\lambda) d \lambda=V(0)-V(t) \geq 0 .
$$

Regarding the Barbalat lemma, i.e., $\lim _{t \rightarrow \infty} \sigma(t)=\lim _{t \rightarrow \infty} \mu|s|=0$, it is clear to note that $\mathrm{s}(\mathrm{t})=0$. As a result, as long as $\mu$ is taken as the positive values, the coverage of the adaptation law is now approved and guaranteed.

Remark 2. While the state variables of the system reach the sliding surfaces, the high frequent control actions should be switched. In fact, this switching leads us to make the chattering phenomenon, which results in the reduction of the useful longevity of the operators. In order to alleviate the chattering phenomenon, the $\operatorname{sign}\left(s_{i}\right)$ in Eq. (11) is replaced with the $\tanh \left(\varepsilon s_{i}\right)$; $(\varepsilon>0)$. Therefore, the final control action becomes as

$$
\begin{aligned}
u_{i}(t)= & m_{i}(x(t))-t_{i}(y(t))+M_{i}(x(t)) \hat{\zeta}-T_{i}(y(t)) \hat{\Gamma} \\
& -\left(\hat{\omega}_{i}+\hat{\beta}_{i}\right) \operatorname{sgn}\left(s_{i}\right)-k_{i} \tanh \left(\varepsilon s_{i}\right) ; i=1,2, \ldots, n .
\end{aligned}
$$

Remark 3. It should be noted that the replace of $\tanh \left(\varepsilon s_{i}\right) ;(\varepsilon>0)$ with $\operatorname{sign}\left(s_{i}\right) ; i=$ $1,2, \ldots, n$, has no effect on the robustness.

Proof. Let us present the sign function by the following

$$
s_{i}=\operatorname{sgn}\left(s_{i}\right)\left|s_{i}\right| \text {. }
$$

Given that $s_{i}$ is not equal to zero, Eq. (31) could be simply rewritten through Eq. (32), i.e.,

$$
\operatorname{sgn}\left(s_{i}\right)=\frac{s_{i}}{\left|s_{i}\right|} \text {. }
$$

Now, for real number $s_{i}$, Eq. (33) could be resulted as

$$
\left|s_{i}\right|=\operatorname{sgn}\left(s_{i}\right) s_{i}
$$

The derivative of the absolute value function for $s_{i} \neq 0$ is taken as the signum function by

$$
\operatorname{sgn}\left(s_{i}\right)=\frac{d\left|s_{i}\right|}{d s_{i}}
$$

By using $H\left(s_{i}\right)$ as the Heaviside step function (using the standard formalism $H(0)=1 / 2$ ), Eq. (35) can be written by

$$
\operatorname{sgn}\left(s_{i}\right)=2 H\left(s_{i}\right)-1 .
$$

Now, it is clear to derive the distributional derivative, since the derivative of the signum function is two times the Dirac delta function, i.e.,

$$
\frac{d \operatorname{sgn}\left(s_{i}\right)}{d s_{i}}=\frac{2 d H\left(s_{i}\right)}{d s_{i}}=2 \delta\left(s_{i}\right) .
$$


It should be noted that the signum can be written through the Iverson bracket notation, i.e.,

$$
\operatorname{sgn}\left(s_{i}\right)=-\left[s_{i}<0\right]+\left[s_{i}>0\right] .
$$

By using $\varepsilon>0$, a smooth approximation of the sign function is taken as

$$
\operatorname{sgn}\left(s_{i}\right) \approx \tanh \left(\varepsilon s_{i}\right) \text {, }
$$

where another approximation could be given by Eq. (39)

$$
\operatorname{sgn}\left(s_{i}\right) \approx \frac{s_{i}}{\sqrt{s_{i}^{2}+\varphi^{2}}} .
$$

This relation gets sharper if $\varphi \rightarrow 0$. In fact, the outcome is the derivative of $\sqrt{s_{i}^{2}+\varphi^{2}}$, while the result is exactly equal for all nonzero $s_{i}$ if $\varphi=0$.

\section{The numerical simulation results}

In order to consider the performance and the applicability of the proposed control approach, a number of numerical simulations are now carried out through MATLAB programming language. The hyper-chaotic Lü system is first supposed to be taken as the master system by the following

$$
\text { Lü }:\left\{\begin{array}{l}
\dot{x_{1}}(t)=a\left(x_{2}(t)-x_{1}(t)\right)+x_{4}(t)+\Delta r_{1}(x(t), t)+d_{1}^{m} \\
\dot{x_{2}}(t)=-x_{1}(t) x_{3}(t)+c x_{2}(t)+\Delta r_{2}(x(t), t)+d_{2}^{m} \\
\dot{x_{3}}(t)=x_{1}(t) x_{2}(t)-\mathrm{b} x_{3}(t)+\Delta r_{3}(x(t), t)+d_{3}^{m} \\
\dot{x_{4}}(t)=x_{1}(t) x_{3}(t)+r x_{4}(t)+\Delta r_{4}(x(t), t)+d_{4}^{m},
\end{array}\right.
$$

where the whole of unknown parameters are taken as $a=36, b=3, c=20$ and $r=1$ to exhibit the hyper-chaotic behaviour. In addition, the Chen system is supposed to be taken as the slave system by the following

$$
\text { Chen: }\left\{\begin{array}{l}
\dot{y_{1}}(t)=p\left(y_{2}(t)-y_{1}(t)\right)+y_{4}(t)+\Delta R_{1}(y(t), t)+d_{1}^{s}+u_{1}(t) \\
\dot{y_{2}}(t)=-y_{1}(t) y_{3}(t)+s y_{2}(t)+t y_{1}(t)+\Delta R_{2}(y(t), t)+d_{2}^{s}+u_{2}(t) \\
\dot{y_{3}}(t)=y_{1}(t) y_{2}(t)-\mathrm{q} y_{3}(t)+\Delta R_{3}(y(t), t)+d_{3}^{s}+u_{3}(t) \\
\dot{y_{4}}(t)=y_{2}(t) y_{3}(t)+z y_{4}(t)+\Delta R_{4}(y(t), t)+d_{4}^{s}+u_{4}(t)
\end{array},\right.
$$

where the whole of unknown parameters are chosen as $p=35, t=7, s=12, q=3$ and $z=-0.5$ to exhibit the hyper-chaotic behaviour. Now, disturbances and uncertainties are added to the master and the slave systems, i.e.,

$$
\begin{aligned}
& \left\{\begin{array} { l } 
{ \Delta r _ { 1 } ( x ( \mathrm { t } ) , t ) = - 2 . 1 0 \operatorname { s i n } ( \pi x _ { 1 } ( \mathrm { t } ) ) } \\
{ \Delta r _ { 2 } ( x ( \mathrm { t } ) , t ) = - 3 . 3 6 \operatorname { s i n } ( 2 . 5 \pi x _ { 2 } ( \mathrm { t } ) ) } \\
{ \Delta r _ { 3 } ( x ( \mathrm { t } ) , t ) = - 5 . 3 6 \operatorname { s i n } ( 2 \pi x _ { 3 } ( \mathrm { t } ) ) } \\
{ \Delta r _ { 4 } ( x ( \mathrm { t } ) , t ) = - 7 . 1 0 \operatorname { s i n } ( 0 . 2 \pi x _ { 4 } ( \mathrm { t } ) ) }
\end{array} \quad \left\{\begin{array}{l}
\Delta R_{1}(y(\mathrm{t}), t)=3.25 \sin \left(2.56 \pi y_{1}(\mathrm{t})\right) \\
\Delta R_{2}(\mathrm{y}(\mathrm{t}), t)=2.36 \sin \left(3.07 \pi y_{2}(\mathrm{t})\right) \\
\Delta R_{3}(y(\mathrm{t}), t)=4.36 \sin \left(1.7 \pi y_{3}(\mathrm{t})\right) \\
\Delta R_{4}(y(\mathrm{t}), t)=1.39 \sin \left(\pi y_{4}(\mathrm{t})\right)
\end{array}\right.\right. \\
& \left\{\begin{array} { l } 
{ d _ { 1 } ^ { m } = - 0 . 2 0 \operatorname { s i n } ( t ) } \\
{ d _ { 2 } ^ { m } = - 0 . 2 0 \operatorname { s i n } ( t ) } \\
{ d _ { 3 } ^ { m } = - 0 . 2 0 \operatorname { s i n } ( t ) } \\
{ d _ { 4 } ^ { m } = - 0 . 2 0 \operatorname { s i n } ( t ) }
\end{array} \quad \left\{\begin{array}{l}
d_{1}^{s}=0.20 \sin (t) \\
d_{2}^{s}=0.20 \sin (t) \\
d_{3}^{s}=0.20 \sin (t) \\
d_{4}^{s}=0.20 \sin (t) .
\end{array}\right.\right.
\end{aligned}
$$


In one such example, the coefficient $\varepsilon$ is taken as 120 . Furthermore, the switching gains, i.e., $k_{1}, k_{2}, k_{3}, k_{4}$ are chosen as 4, 6, 8 and 10, respectively. Regarding Eq. (7), the system error dynamics could be acquired by

$$
\left\{\begin{array}{c}
\dot{e}_{1}(t)=p\left(y_{2}(t)-y_{1}(t)\right)+y_{4}(t)+3.25 \sin \left(2.56 \pi y_{1}(t)\right)+0.2 \sin (\mathrm{t}) \\
\left.-\left(a\left(x_{2}(t)-x_{1}(t)\right)+x_{4}(t)\right)-2.1 \sin \left(\pi x_{1}(\mathrm{t})\right)-0.2 \sin (\mathrm{t})\right)+u_{1}(t) \\
\dot{e}_{2}(t)=t y_{1}(t)-y_{1}(t) y_{3}(t)+s y_{2}(t)+2.36 \sin \left(3.07 \pi y_{2}(t)\right)+0.2 \sin (\mathrm{t}) \\
\quad+\left(x_{1}(t) x_{3}(t)-c x_{2}(t)+3.36 \sin \left(2.5 \pi x_{2}(\mathrm{t})\right)+0.2 \sin (\mathrm{t})\right)+u_{2}(t) \\
\dot{e}_{3}(\mathrm{t})=y_{1}(t) y_{2}(t)-q y_{3}(t)+4.36 \sin \left(1.7 \pi y_{3}(t)\right)+0.2 \sin (\mathrm{t}) \\
\quad-\left(x_{1}(t) x_{2}(t)-b x_{3}(t)-5.36 \sin \left(2 \pi x_{3}(\mathrm{t})\right)-0.2 \sin (\mathrm{t})\right)+u_{3}(t) \\
\dot{e}_{4}(t)=y_{2}(t) y_{3}(t)+z y_{4}(t)+1.39 \sin \left(\pi y_{4}(t)\right)+0.2 \sin (\mathrm{t}) \\
\quad-\left(x_{1}(t) x_{3}(t)+r x_{4}(t)-7.1 \sin \left(0.2 \pi x_{4}(\mathrm{t})\right)-0.2 \sin (\mathrm{t})\right)+u_{4}(t) .
\end{array}\right.
$$

Afterwards, the sliding surfaces are all chosen as $s_{1}=4 e_{1}, s_{2}=4 e_{2}, s_{3}=4 e_{3}$ and $s_{4}=$ $4 e_{4}$, respectively. Subsequently, in accordance with Eq. (11), the control actions are all taken as

$$
\left\{\begin{array}{l}
u_{1}(t)=\left(\hat{a}\left(x_{2}(t)-x_{1}(t)\right)+x_{4}(t)\right)-\hat{p}\left(y_{2}(t)-y_{1}(t)\right)-y_{4}(t)-\left(\hat{\omega}_{1}+\hat{\beta}_{1}\right) \operatorname{sgn}\left(s_{1}\right)-4 \tanh \left(480 e_{1}(t)\right) \\
u_{2}(t)=\left(-x_{1}(t) x_{3}(t)+\hat{c} x_{2}(t)\right)-\hat{t} y_{1}(t)+y_{1}(t) y_{3}(t)-\hat{s} y_{2}(t)-\left(\hat{\omega}_{2}+\hat{\beta}_{2}\right) \operatorname{sgn}\left(s_{2}\right)-6 \tanh \left(480 \mathrm{e}_{2}(t)\right) \\
u_{3}(t)=\left(x_{1}(t) x_{2}(t)-\hat{b} x_{3}(t)\right)-y_{1}(t) y_{2}(t)+\hat{q} y_{3}(t)-\left(\hat{\omega}_{3}+\hat{\beta}_{3}\right) \operatorname{sgn}\left(s_{3}\right)-8 \tanh \left(480 e_{3}(t)\right) \\
u_{4}(t)=\left(x_{1}(t) x_{3}(t)+\hat{r} x_{4}(t)\right)-y_{2}(t) y_{3}(t)-\hat{z} y_{4}(t)-\left(\hat{\omega}_{4}+\hat{\beta}_{4}\right) \operatorname{sgn}\left(s_{4}\right)-10 \tanh \left(480 e_{4}(t)\right) .
\end{array}\right.
$$

Now, the initial conditions of the Lü and the Chen systems are taken as $x_{1}(0)=-2, x_{2}(0)=$ $7.5, x_{3}(0)=-8, x_{4}(0)=0.05 ; y_{1}(0)=7, y_{2}(0)=4, y_{3}(0)=-1.25, y_{4}(0)=6.5$, in the experiment 1, respectively. Moreover, the adaptation laws; Eq. (12), has been added to Eq. (44). The present time response of the adaptation vector parameters, i.e., $\hat{\omega}, \hat{\beta}, \hat{\zeta}$ and $\hat{\Gamma}$ are depicted in figures 2 to 5 . Obviously, the whole of the present adaptation parameters converge to be constant after a reasonable amount of time. In this numerical example, figures 6 to 9 illustrate the states of the master and the slave systems, while figures 10 to 13 illustrate the control actions generated through the proposed control scheme. In one such case, synchronization errors of the hyperchaotic Lü and hyper-chaotic Chen systems are all illustrated in figures 14 to 17 that converge to be zero, as are easily obvious.

In order to consider the applicability of the approach proposed here, the initial conditions of the Lü and the Chen systems are reassigned as $x_{1}(0)=-0.75, x_{2}(0)=4.5, x_{3}(0)=$ $-4, x_{4}(0)=0.1 ; y_{1}(0)=3.5, y_{2}(0)=2, y_{3}(0)=-0.5, y_{4}(0)=3.5$, in the second experiment, respectively. The present time response of the adaptation vector parameters, i.e. $\hat{\omega}, \hat{\beta}, \zeta$ and $\hat{\Gamma}$ are only depicted in figures 18 to 21 . Obviously, the whole of the present adaptation parameters converge to be constant after a desirable amount of time. In order to present the investigated results in the concise form, the reset parameters of the approach proposed here, which are all shown in the first experiment, are not illustrated.

Finally, the initial conditions of the Lü and the Chen systems are reassigned one more time, in the third experiment, as $x_{1}(0)=0.05, x_{2}(0)=13.75, x_{3}(0)=3.25, x_{4}(0)=4 ; y_{1}(0)=$ $-0.25, y_{2}(0)=8.25, y_{3}(0)=2.5, y_{4}(0)=7.5$, respectively. With this purpose, the present time response of the adaptation vector parameters, i.e., $\hat{\omega}, \hat{\beta}, \hat{\zeta}$ and $\hat{\Gamma}$ are only shown in figures 22 to 25 . Moreover, the whole of the present adaptation parameters converge to be constant after a reasonable amount of time. The reset parameters of the approach proposed are the same as the second experiment, i.e., all of them are not illustrated. 


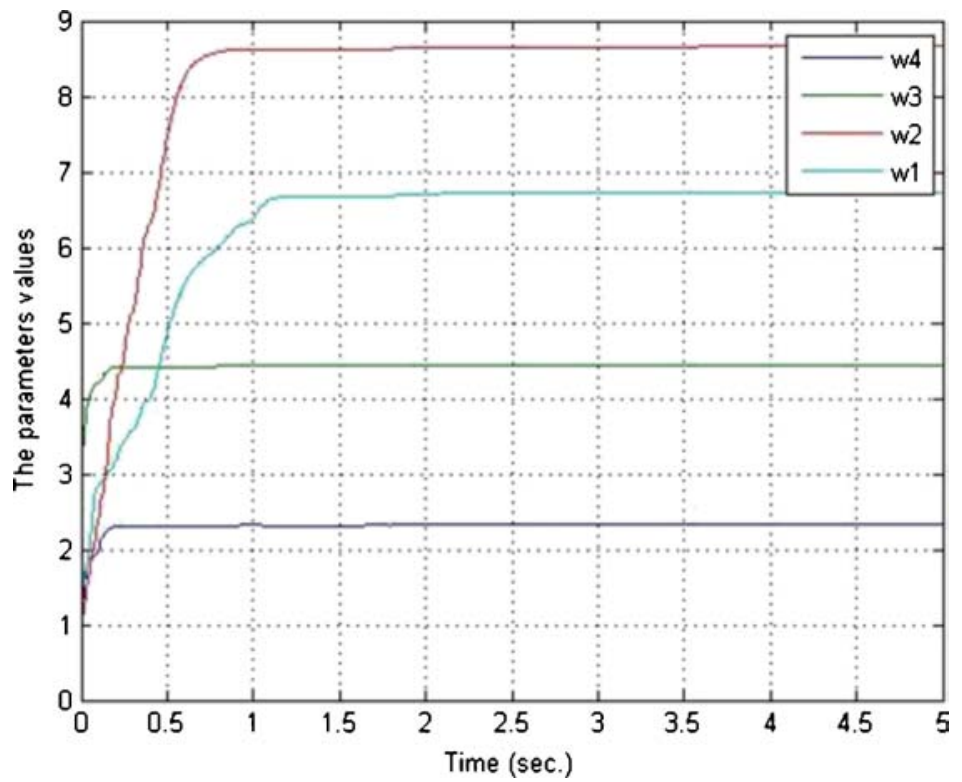

Figure 2. The adaptation vector parameters, i.e., $\left(\hat{\omega}_{i}\right) ; i=1,2,3,4$ in the experiment 1 .

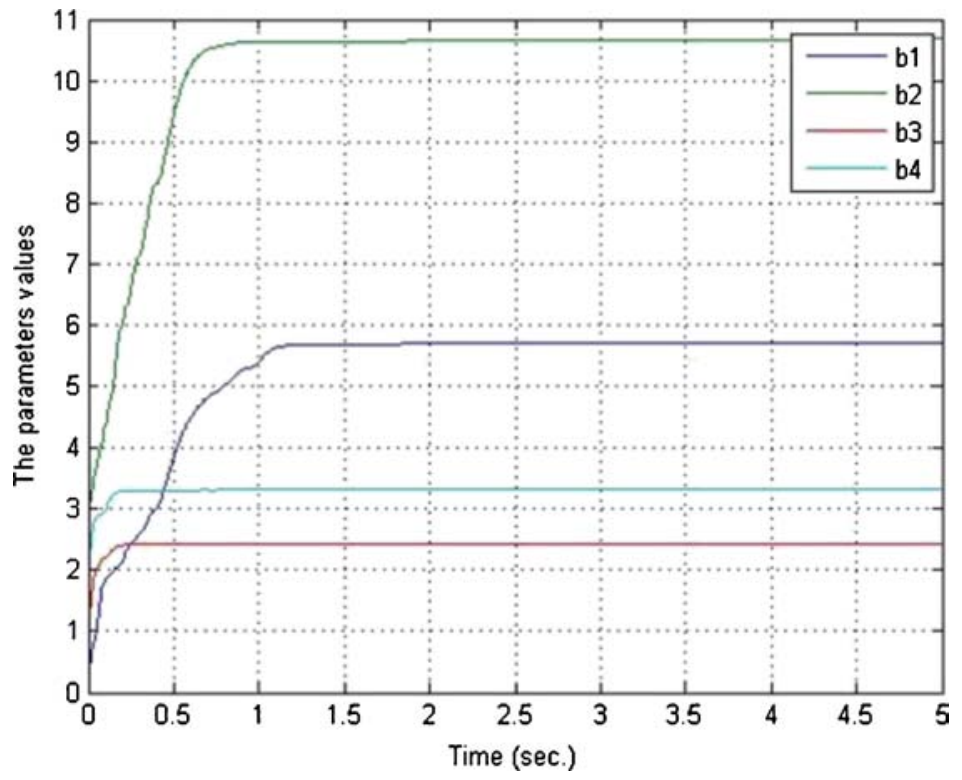

Figure 3. The adaptation vector parameters, i.e., $\left(\hat{\beta}_{i}\right) ; i=1,2,3,4$ in the experiment 1 . 


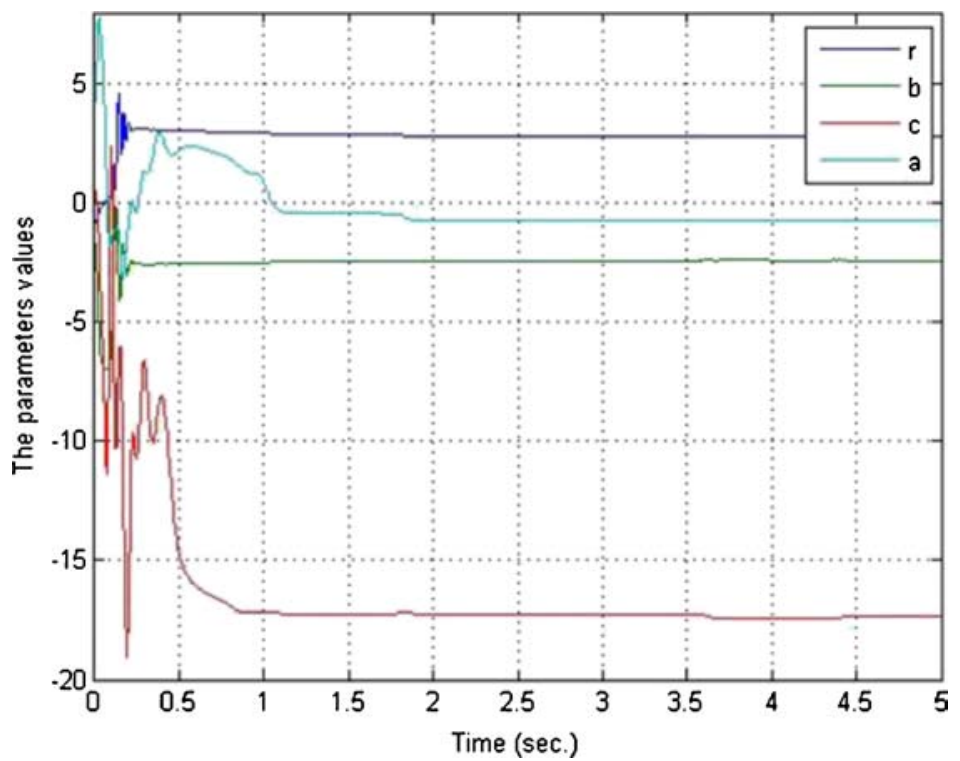

Figure 4. The adaptation vector parameters, i.e., $\hat{\zeta}$ in the experiment 1.

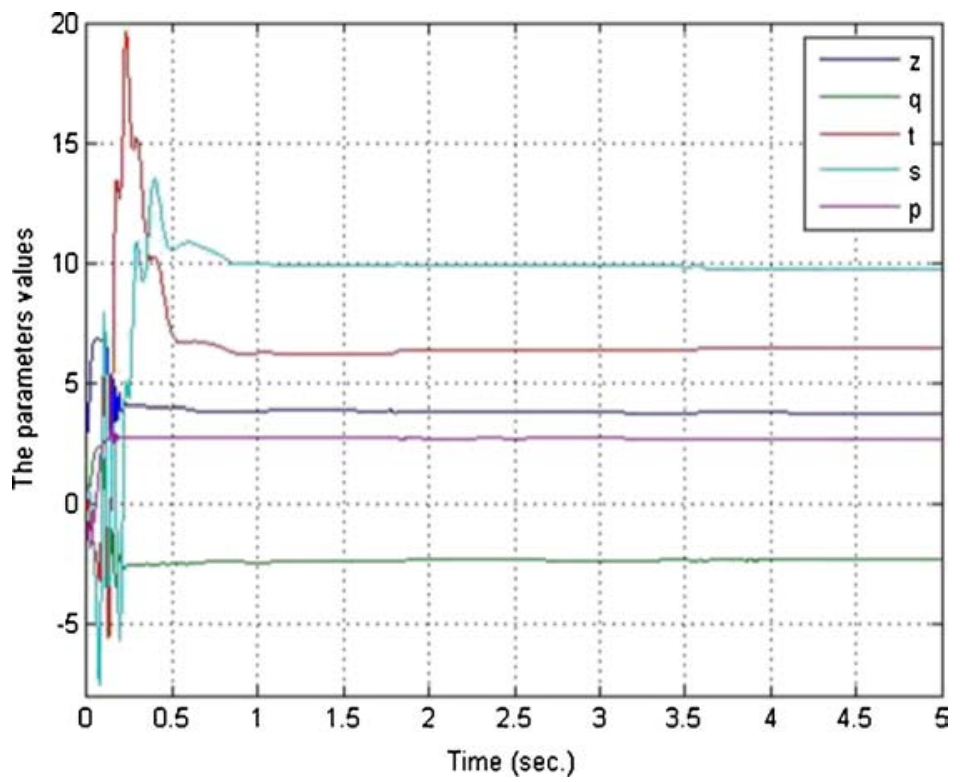

Figure 5. The adaptation vector parameters, i.e., $\hat{\Gamma}$ in the experiment 1 . 


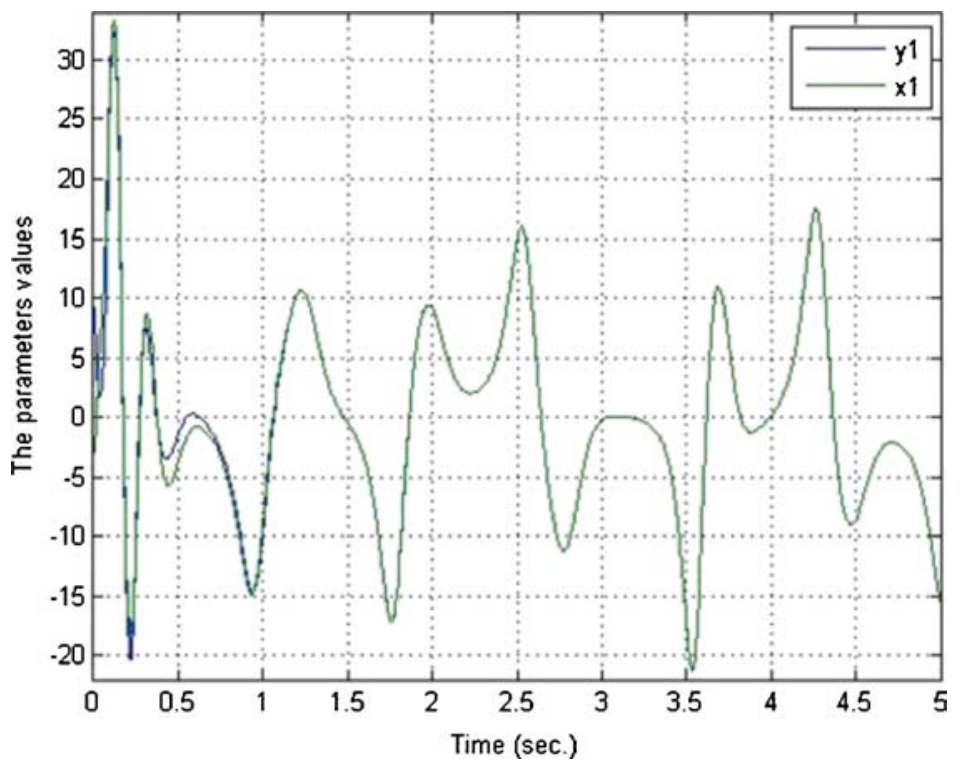

Figure 6. The first states of the master (x1) and slave (y1) systems in the experiment 1 .

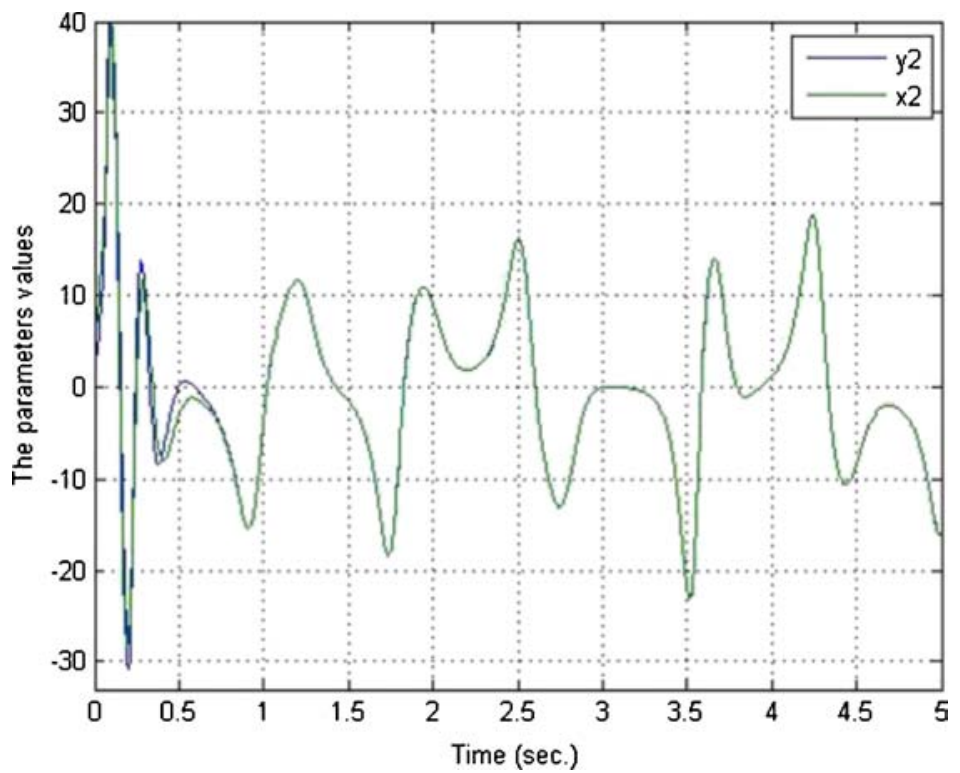

Figure 7. The second states of the master (x2) and slave (y2) systems in the experiment 1 . 


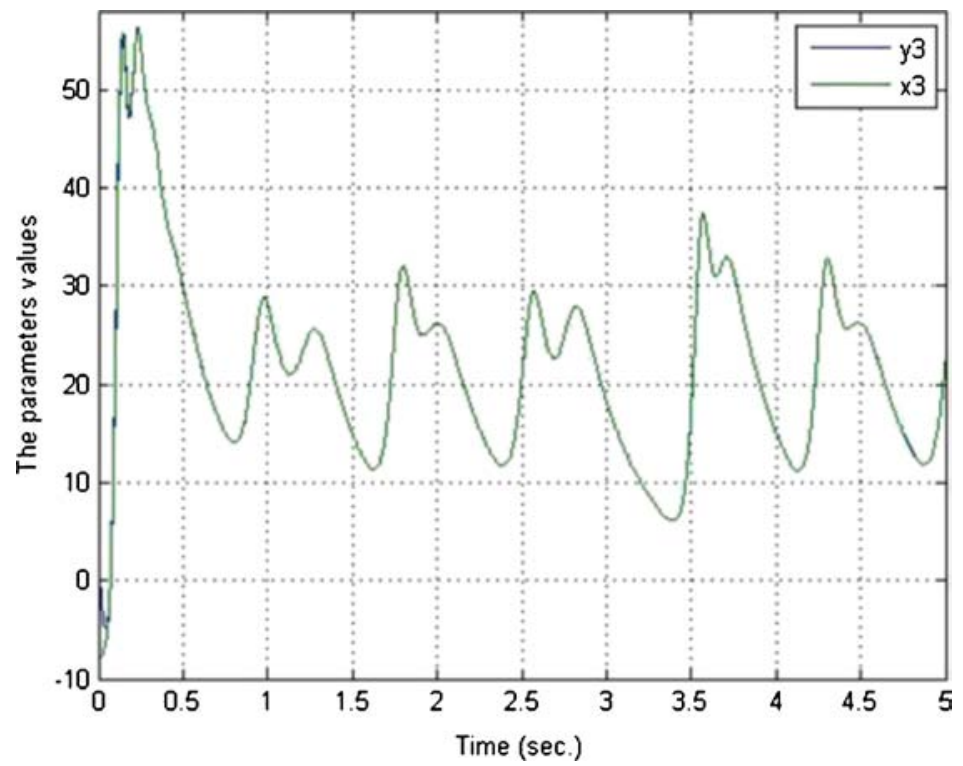

Figure 8. The third states of the master (x3) and slave (y3) systems in the experiment 1 .

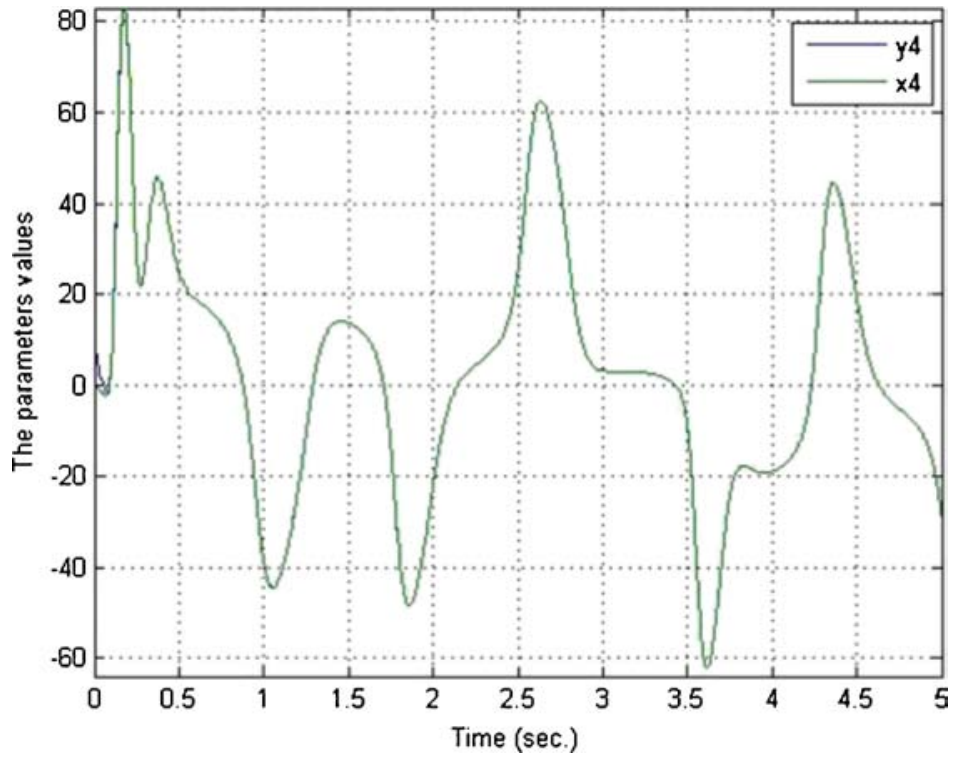

Figure 9. The fourth states of the master $(\mathrm{x} 4)$ and slave $(\mathrm{y} 4)$ systems in the experiment 1 . 


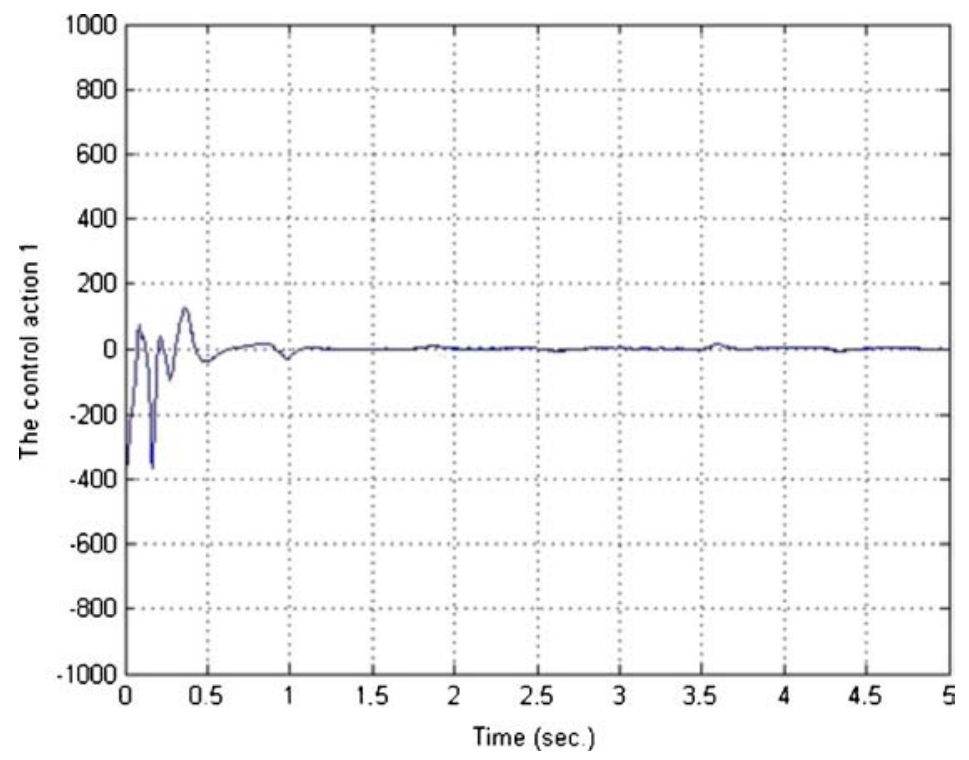

Figure 10. The first control action generated through the proposed approach in the experiment 1 .

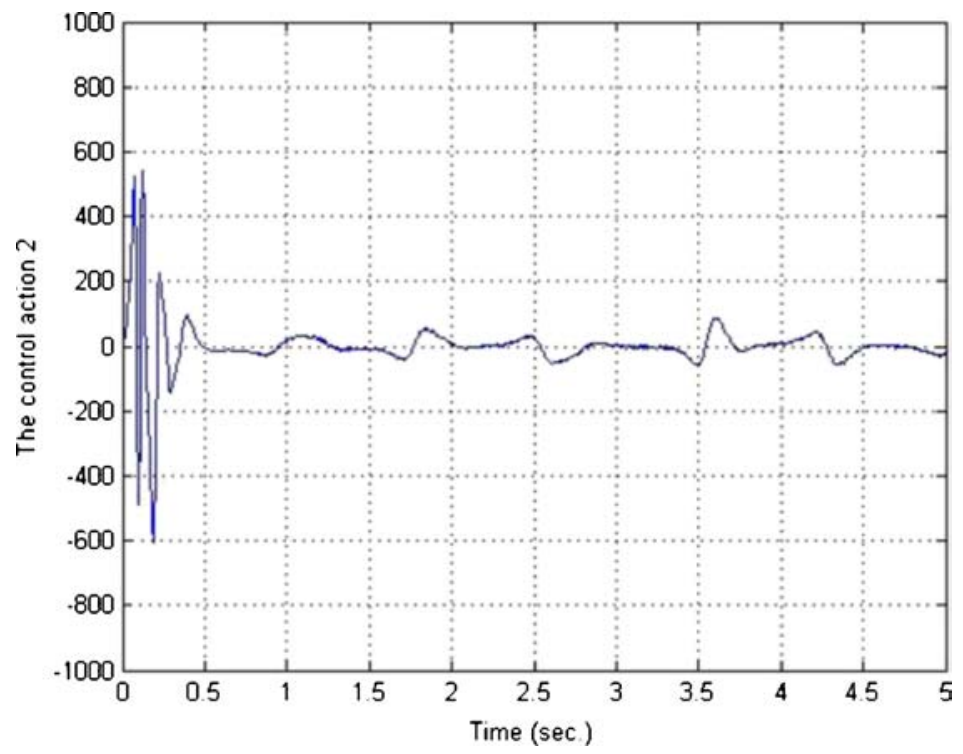

Figure 11. The second control action generated through the proposed approach in the experiment1. 


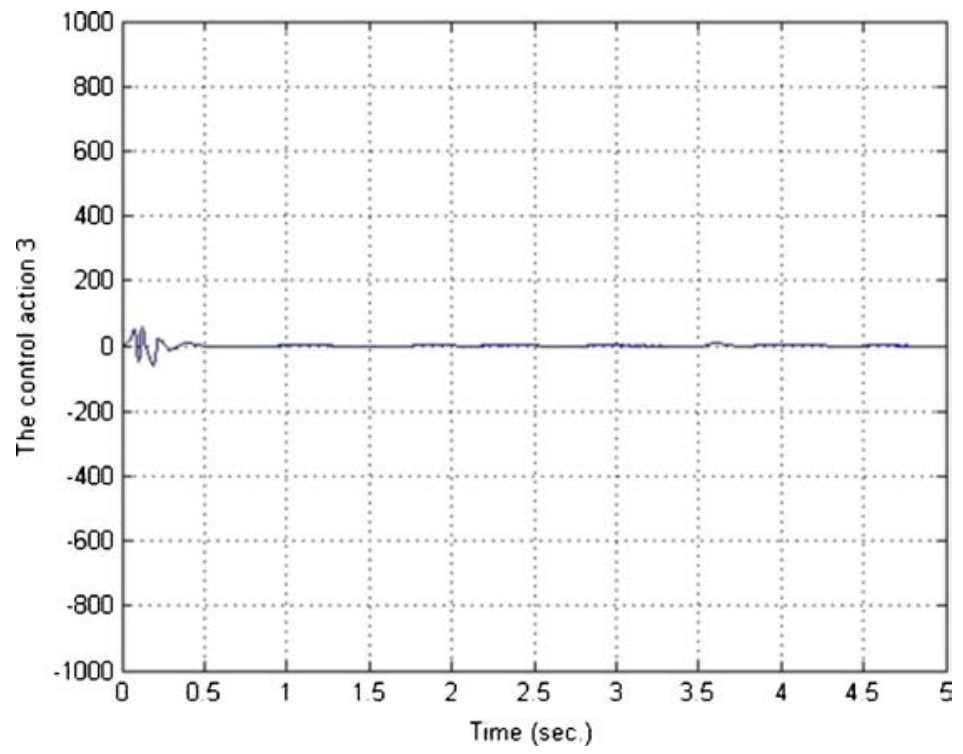

Figure 12. The third control action generated through the proposed approach in the experiment 1 .

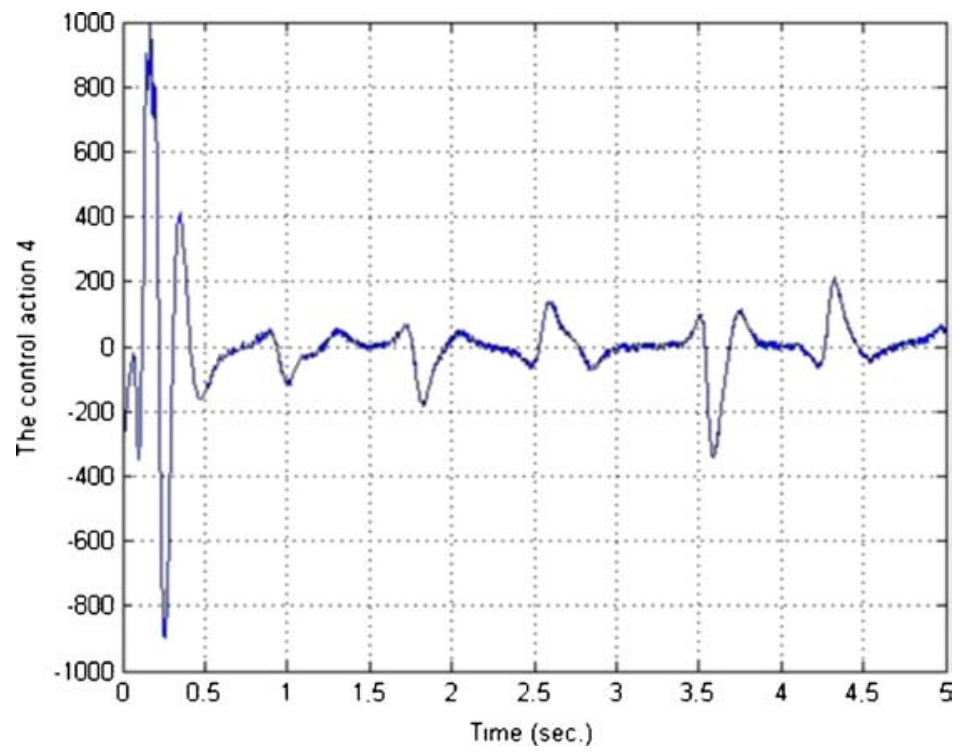

Figure 13. The fourth control action generated through the proposed approach in the experiment 1 . 


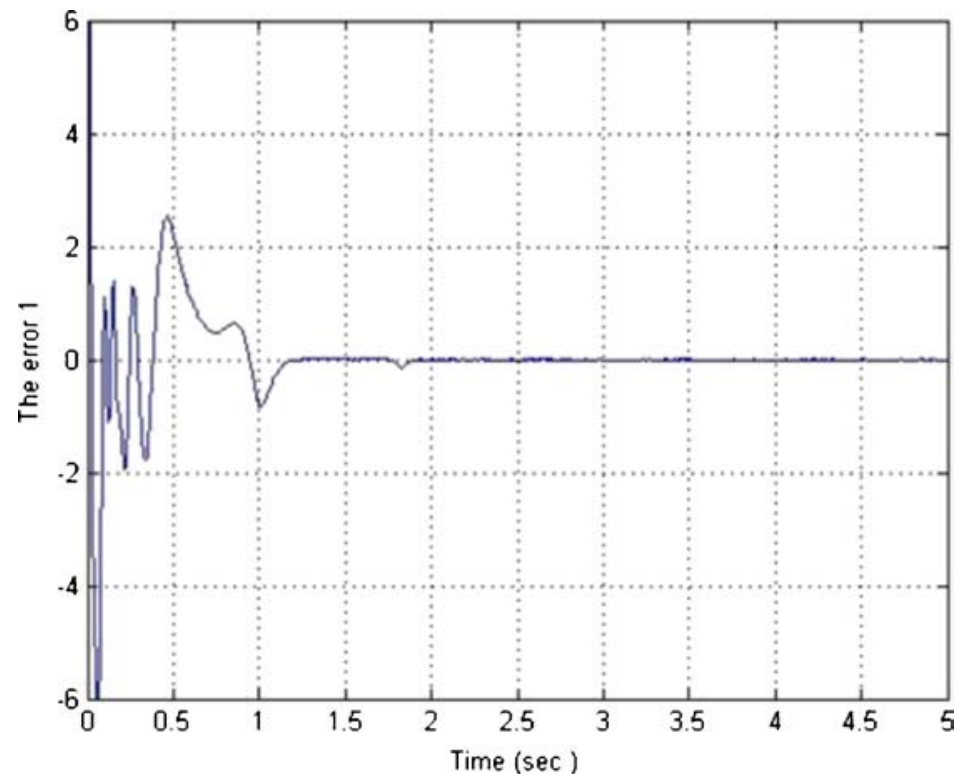

Figure 14. The first synchronization error between the hyper-chaotic Lü and Chen systems in the experiment 1 .

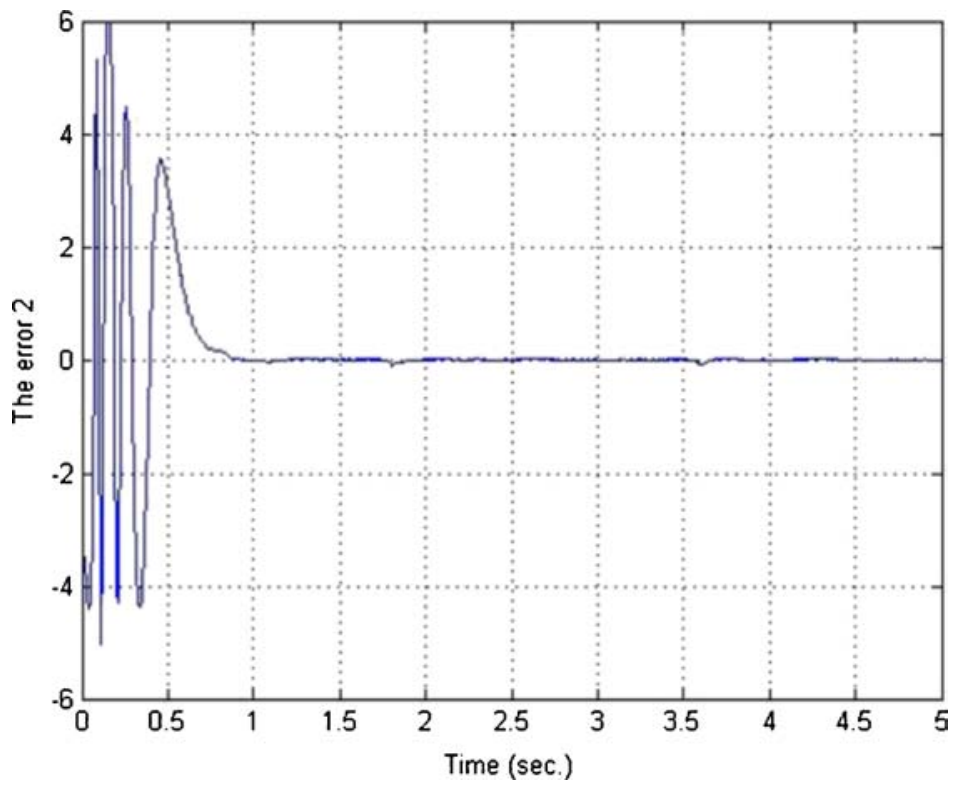

Figure 15. The second synchronization error between the hyper-chaotic Lü and Chen systems in the experiment 1 . 


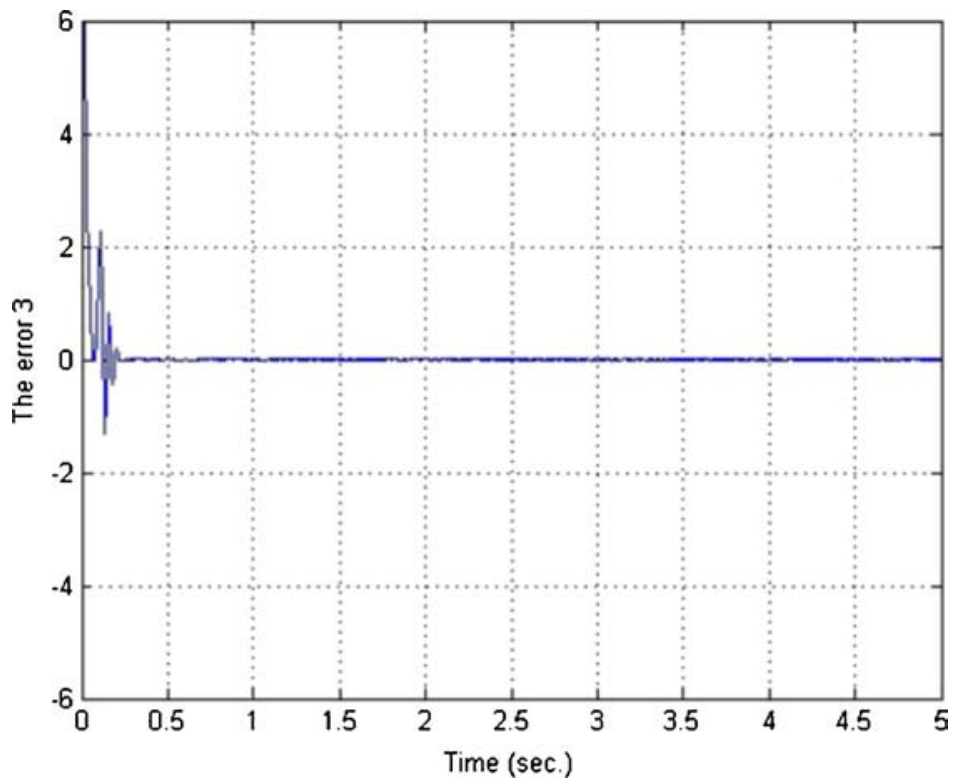

Figure 16. The third synchronization error between the hyper-chaotic Lü and Chen systems in the experiment 1 .

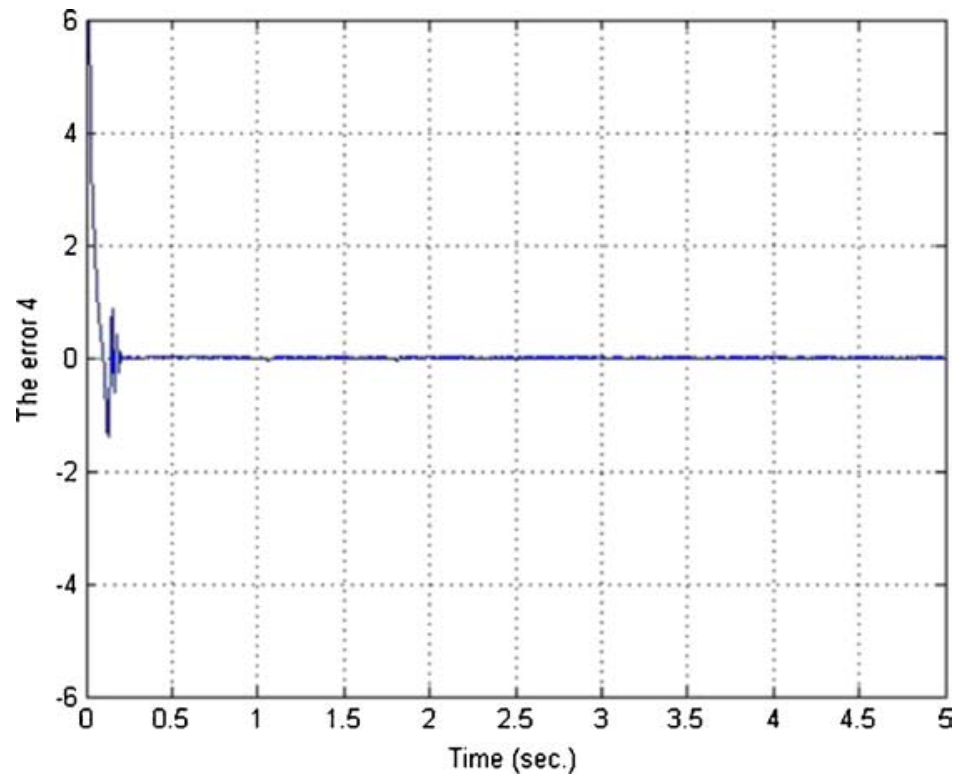

Figure 17. The fourth synchronization error between the hyper-chaotic Lü and Chen systems in the experiment 1 . 


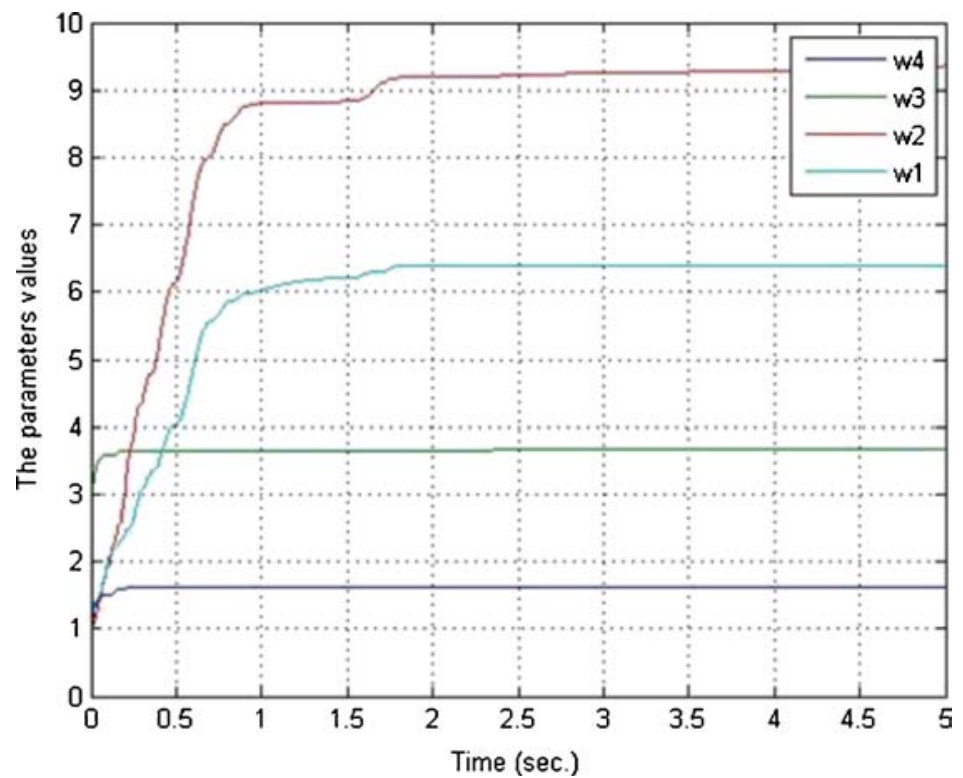

Figure 18. The adaptation vector parameters, i.e., $\hat{\omega}_{i} ; i=1,2,3,4$ in the experiment 2 .

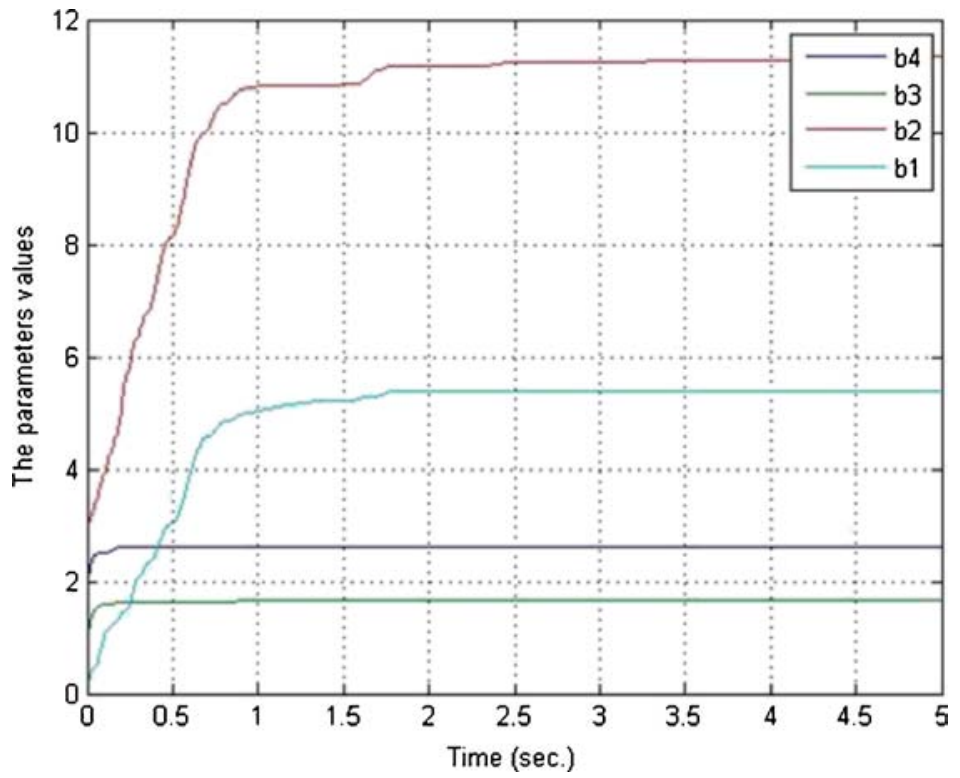

Figure 19. The adaptation vector parameters, i.e., $\hat{\beta}_{i} ; i=1,2,3,4$ in the experiment 2 . 


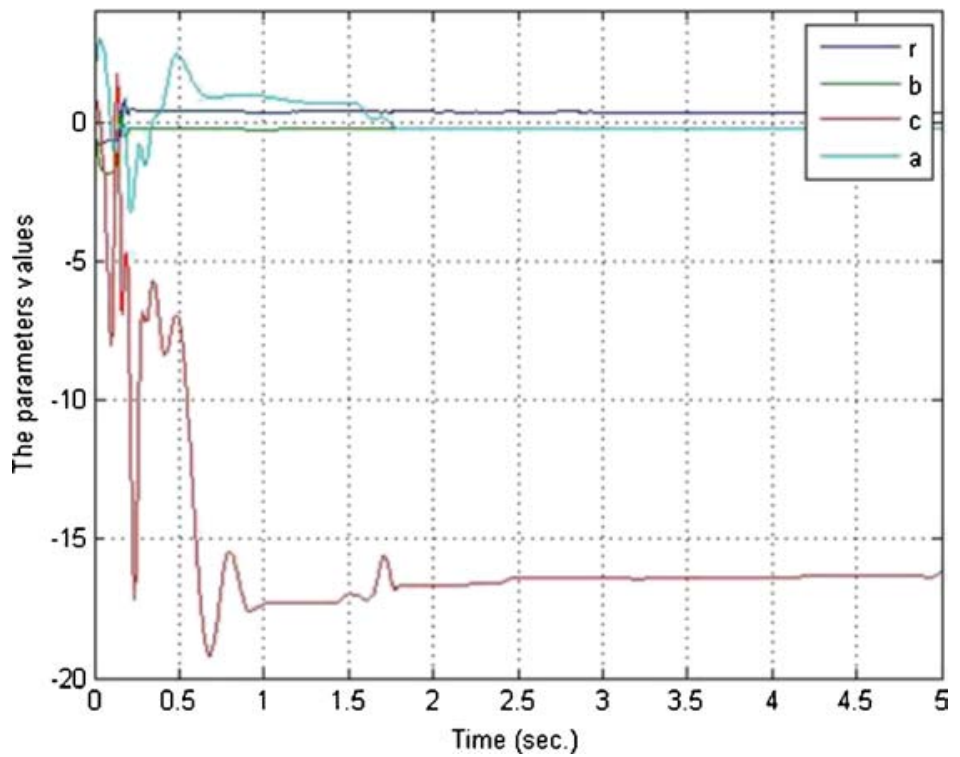

Figure 20. The adaptation vector parameters, i.e., $\hat{\zeta}$ in the experiment 2 .

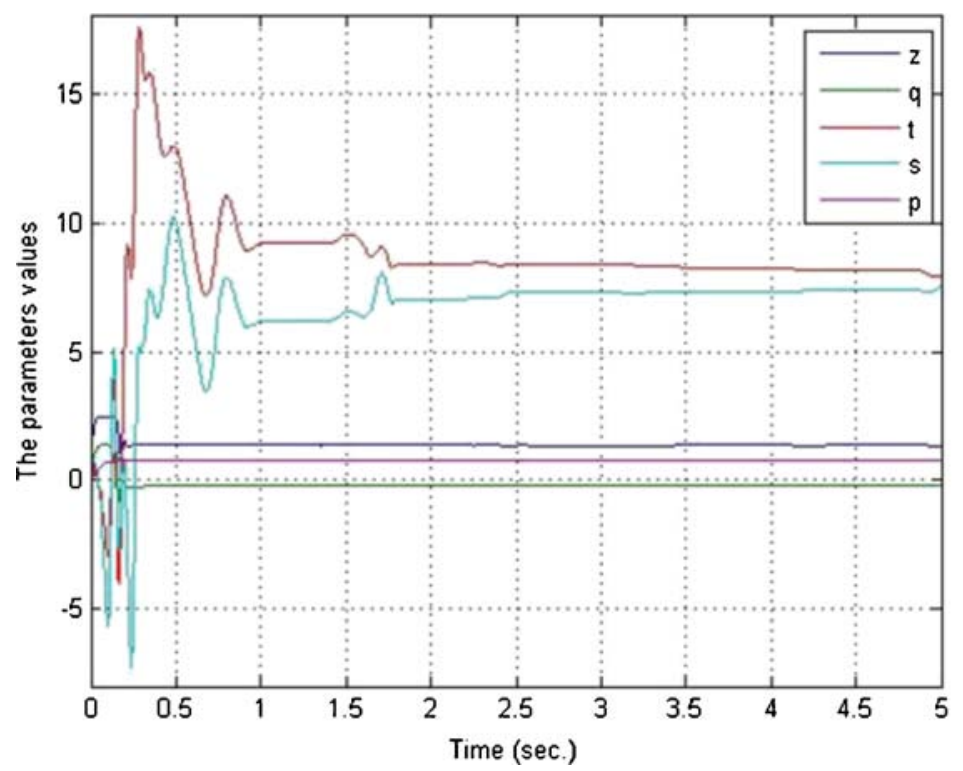

Figure 21. The adaptation vector parameters, i.e., $\hat{\Gamma}$ in the experiment 2. 


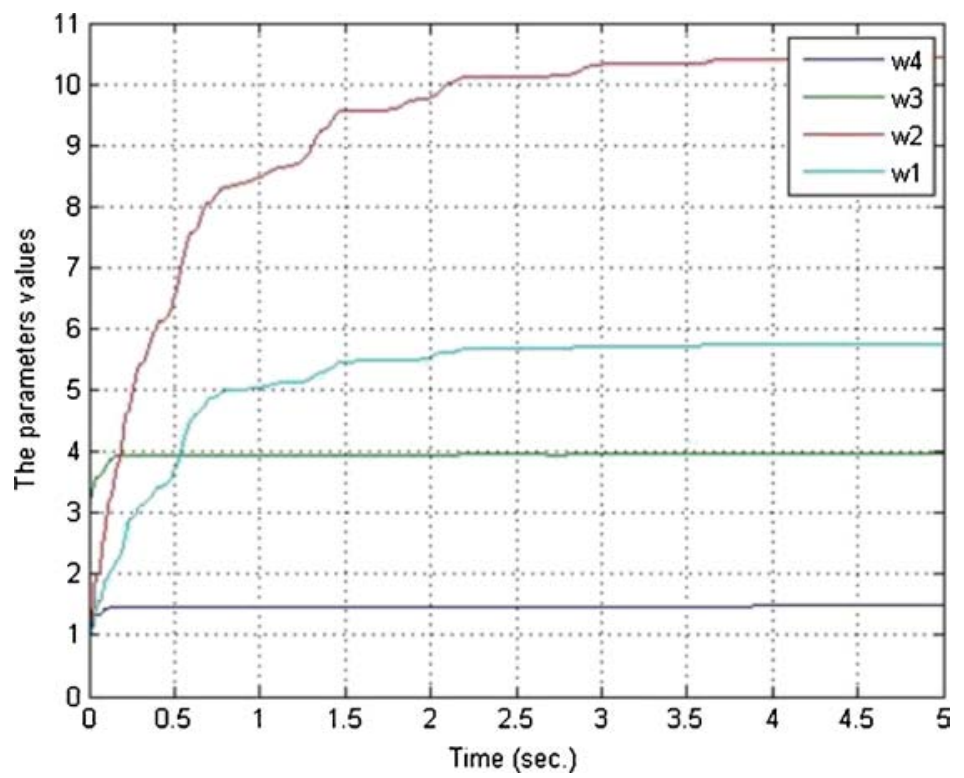

Figure 22. The adaptation vector parameters, i.e., $\left(\hat{\omega}_{i}\right) ; i=1,2,3,4$ in the experiment 3 .

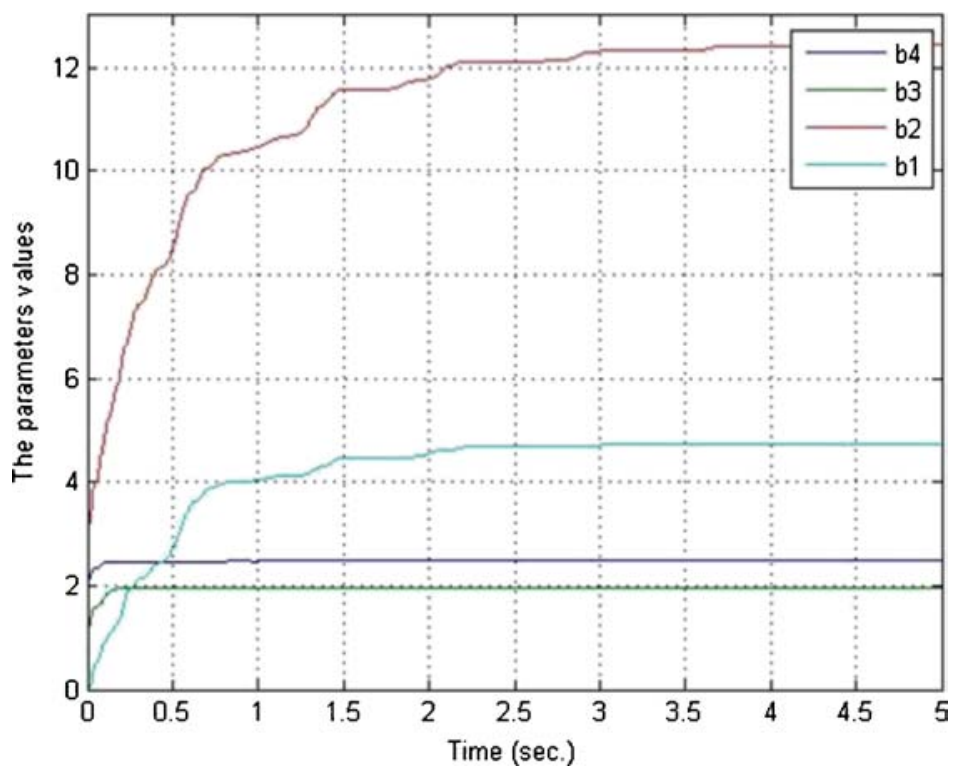

Figure 23. The adaptation vector parameters, i.e., $\hat{\beta}_{i} ; i=1,2,3,4$ in the experiment 3 . 


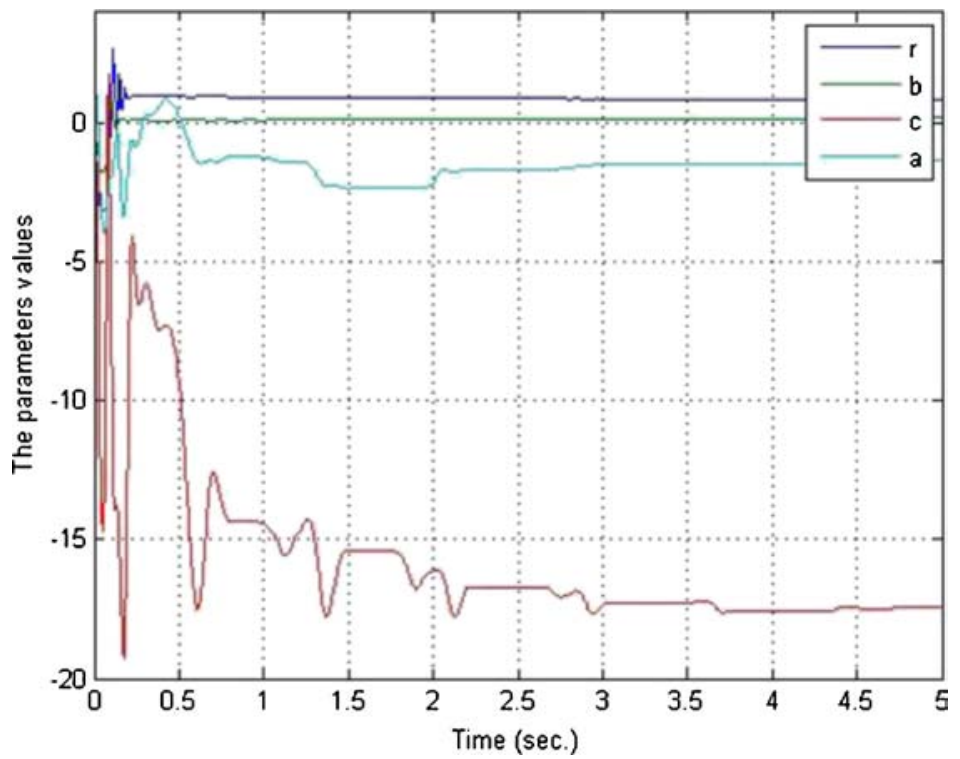

Figure 24. The adaptation vector parameters, i.e., $\hat{\zeta}$ in the experiment 3 .

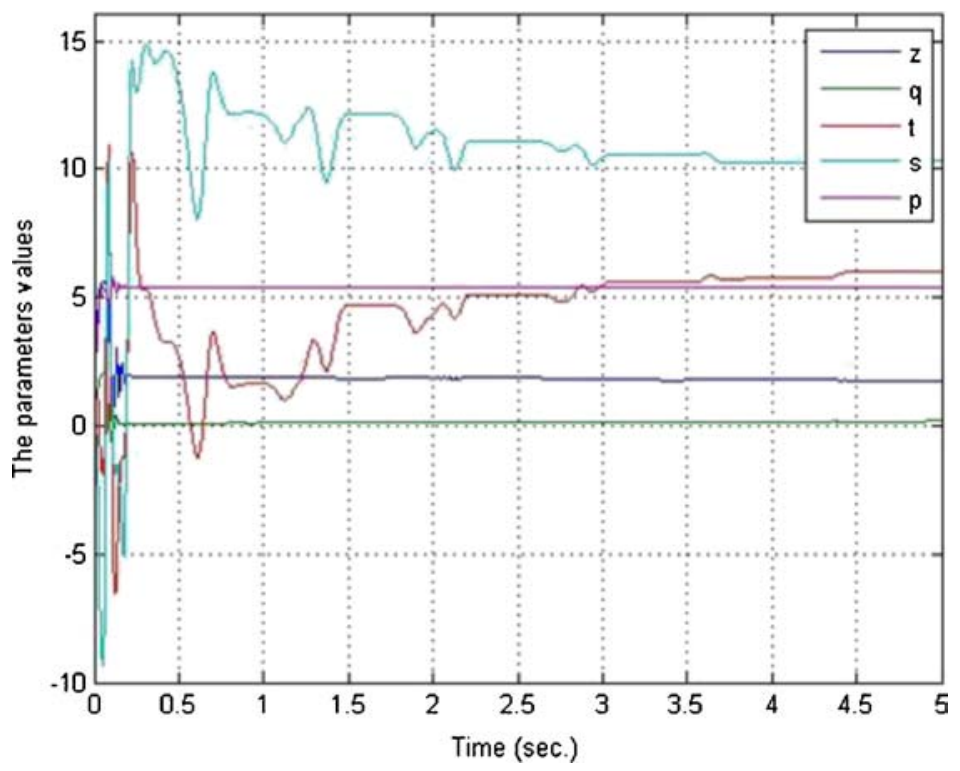

Figure 25. The adaptation vector parameters, i.e., $\hat{\Gamma}$ in the experiment 3 . 


\section{Conclusions}

An efficient control scheme has been investigated in the present research to synchronize two non-identical hyper-chaotic master/slave systems, which are taken into consideration as the models of so many simple and complicated processes. This idea is based on the realization of the adaptive sliding mode control approach. The present investigation is applied to the master and the slave systems, since both of them are encountered uncertainties, disturbances and unknown parameters. The acquired numerical simulation results demonstrate the accuracy of the proposed control scheme.

\section{Acknowledgements}

We thank the reviewers for useful comments. Our sincere thanks are due to the Islamic Azad University (IAU) for the support of research work.

\section{References}

Abdullah Ali 2013 Synchronization and secure communication of uncertain chaotic systems based on fullorder and reduced-order output-affine observers. Appl. Mathematics and Computation 219(19): 1000010011

Agrawal S K, Srivastava M and Das S 2012 Synchronization of fractional order chaotic systems using active control method. Chaos, Solitons \& Fractals 45(6): 737-752

Chi-Ching Yang 2012 Synchronization of second-order chaotic systems via adaptive terminal sliding mode control with input nonlinearity. J. Franklin Institute 349(6): 2019-2032

Guo Rongwei 2012 Finite-time stabilization of a class of chaotic systems via adaptive control method. Communications in Nonlinear Science and Numerical Simulation 17(1): 255-262

Kuntanapreeda Suwat and Sangpet Teerawat 2012 Synchronization of chaotic systems with unknown parameters using adaptive passivity-based control. J. Franklin Institute 349(8): 2547-2569

Lorenz E N 1963 Deterministic nonperiodic flows. J. Atmospheric Sci. 20: 130-140

Razminia Abolhassan and Baleanu Dumitru 2013 Complete synchronization of commensurate fractional order chaotic systems using sliding mode control. Mechatronics 23(7): 873-879

Rossler O E 1979 An equation for hyper chaos. Phys. Lett. A 71: 155-157

Yang Junqi, Chen Yantao and Zhu Fanglai 2014 Singular reduced-order observer-based synchronization for uncertain chaotic systems subject to channel disturbance and chaos-based secure communication. Appl. Mathematics and Computation 229(25): 227-238

Ye Zhiyong and Deng Cunbing 2012 Adaptive synchronization to a general non-autonomous chaotic system and its applications. Nonlinear Analysis, Real World Applications 13(2): 840-849

Yin Chun, Dadras Sara and Zhong Shou-ming 2012 Design an adaptive sliding mode controller for driveresponse synchronization of two different uncertain fractional-order chaotic systems with fully unknown parameters. J. Franklin Institute 349(10): 3078-3101

Yuan Guohui, Zhang Xin and Wang Zhuoran 2014 Generation and synchronization of feedback-induced chaos in semiconductor ring lasers by injection-locking. Optik - Int. J. Light and Electron Opt. 125(8): 1950-1953

Zhao Jiakun 2012 Adaptive Q-S synchronization between coupled chaotic systems with stochastic perturbation and delay. Appl. Mathematical Modelling 36(7): 3312-3319 